\title{
THE CHIRAL APPROACH TO THE ELECTROWEAK INTERACTIONS*
}

\author{
F. Feruglio \\ Dipartimento di Fisica, Univ. di Padova \\ I.N.F.N., Sezione di Padova
}

\begin{abstract}
The effective lagrangian approach is reviewed and applied to the case of electroweak interactions.
\end{abstract}

DFPD 92/TH/50

September 1992

${ }^{*}$ Lectures given at the

$2^{\text {nd }}$ National Seminar of Theoretical Physics

Parma, 1-12 September 1992. 


\section{Contents}

$\begin{array}{lll}1 & \text { Introduction } & 1\end{array}$

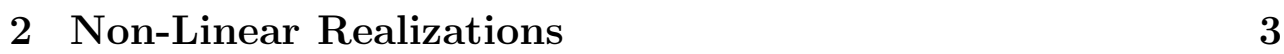

3 Electroweak Interactions: the Lowest Order 10

4 Precision Measurements and Electroweak Radiative cor$\begin{array}{ll}\text { rections } & 17\end{array}$

5 Electroweak Interactions: Higher Orders 24

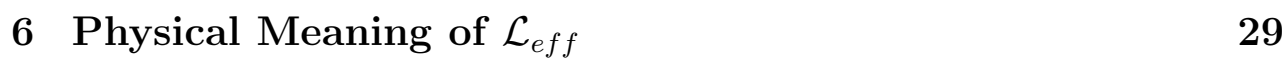

\begin{tabular}{|lll}
\hline & The Matching Conditions & 33
\end{tabular} 


\section{Introduction}

The aim of this course is to provide an introduction to the basic features and methods of effective field theories, working out, as an example, the case of electroweak interactions.

The use of effective lagrangians dates back to the early 60's with the introduction of the non-linear $\sigma$-model [1] as an effective model for low energy strong interactions, explicitly exhibiting chiral symmetry breakdown. The theoretical basis of chiral lagrangians was later formulated by Weinberg [2] in an attempt to characterize the most general S-matrix elements compatible with chiral symmetry and the general requirements of analyticity, unitarity and cluster decomposition property.

Chiral lagrangians are essentially tailored to describe the phenomenon of spontaneous symmetry breaking, which plays a major role in both strong and electroweak interactions. They can be regarded as the low energy limit of an underlying fundamental theory. In the case of strong interactions such a regime is difficult to discuss in the framework of QCD since the knowledge of the non-perturbative dynamical effects is required. Therefore, it is not surprising that in the context of strong interactions the chiral lagrangian approach has found its greatest development [3, 4, 5].

More recently, effective field theories have received attention in the analysis of radiative corrections for electroweak theories [6, 7, 8, 9, 10]. Indeed, in the standard model of electroweak interactions, low energy effective lagrangians naturally occur when some of the particles of the theory become very heavy. Much interest has been devoted to the possibility of an heavy Higgs [11, 12, 13], which is central in the discussion of the electroweak symmetry breaking itself [14].

Interesting problems, related to the non-applicability of the decoupling theorem [15], are also raised by the possibility of heavy fermions [16, 17, 18, 19]. In particular, the case of an heavy top quark calls for the use of the whole apparatus of non-linear realizations, the usual linear realization of the electroweak symmetry being destroyed from the beginning [18]. By removing heavy fermions from the low energy spectrum, one has also to take care of possible anomalies in the gauge currents [20]. These anomalies are consistently avoided thanks to the presence of a Wess-Zumino term 21] in the effective action and to the very specific interplay of the latter with the non-linear realization of the symmetry.

Another fruitful application is that when the fundamental theory one is dealing with is not known, or not precisely defined (actually, this was the case with the nonlinear $\sigma$-model). If the possible symmetries are known, then chiral lagrangians may be naturally introduced. This happens, for instance, with technicolor theories [22], where a considerable amount of information can be extracted from the analysis of the 
corresponding low energy models [23, 6, 0]. Chiral lagrangians are characterized by an infinite tower of non-renormalizable operators, actually all the operators which are consistent with the assumed symmetries. To make practical use of such an infinite expansion one has to be able to select the most relevant terms. In some cases, when the full underlying theory is given, the size of the possible terms can be computed, at least in principle, by matching the predictions of the fundamental field theory and those of the effective one, at some reference scale [24]. This procedure, however, may not be always viable. In this case, some insight about the relevance of the various terms can be obtained by a judicious use of dimensional analysis [25].

An interesting development of the effective lagrangian approach was offered by the study of the hidden gauge symmetries [26] possessed by chiral models. Applications of these symmetries have been made in low energy strong interactions, to describe the lowest spin 1 states [27], and in the study of the so-called strong electroweak sector, to analyze the effects of possible vector resonances [28]. The strongly interacting symmetry breaking sector has been extensively studied by means of chiral lagrangian techniques [29].

Effective field theories can also be introduced to perform a model independent analysis of $S U(2)_{L} \otimes U(1)_{Y}$ supersymmetric gauge theories [30]. Finally, the case of $\mathrm{N}=1$ supergravity [31] shows a certain analogy with the examples quoted above, if interpreted as an effective low energy theory derived by integrating out the massive modes of superstring theories [32].

The lectures are organized as follows. Section 2 contains a review of the nonlinear realizations of a Lie group. The formalism is then applied to the case of weak interactions, whose lowest order lagrangian is derived in section 3. Section 4 presents a simplified discussion of the electroweak radiative corrections, focussing on the LEP I physics. In section 5, the effective electroweak lagrangian is extended to account for quantum effects from heavy particles, and the physical content of the theory is detailed in section 6 . Finally section 7 provides an example of matching between low energy and high energy physics, leading to a specification of the parameters in the effective lagrangian. 


\section{Non-Linear Realizations}

This section contains a short review of the non-linear realizations of a Lie group $G$ [33. We consider a real analytic manifold $M$, together with a Lie Group $G$ 0 of transformations acting on $M$ :

$$
x \rightarrow g x \quad x \in M, \quad g \in G
$$

We assume that there is a special point of $M$ called origin, described by the null vector 0 and invariant under the action of a continuous subgroup $H$ of $G$ :

$$
h 0=0 \quad h \in H
$$

The physical situation one has in mind is that of a manifold of scalar fields with the origin describing the vacuum configuration. The group $G$ is the (global or local) invariance group of the theory and the subgroup $H$ is the invariance group of the vacuum. In other words, one is dealing with the spontaneous breaking of $G$ into $H$.

Our purpose is to characterize all possible non-linear realizations of the group $G$ on the manifold $M$, that is to classify all possible theories corresponding to that pattern of symmetry breaking.

Preliminary to the analysis of this problem is a general result of quantum field theory establishing the independence of the physical content of a theory from the choice of interpolating fields [34]. In the framework of a lagrangian formulation of the theory, the theorem can be rephrased in the following form [35.

- Theorem: If a theory is defined by the lagrangian density:

$$
\mathcal{L}=\mathcal{L}\left(\phi, \partial_{\mu} \phi\right)
$$

depending on a set of scalar fields $\phi$, and if the following local transformation of fields is performed:

$$
\phi=F\left(\phi^{\prime}\right)
$$

then the transformed lagrangian density:

$$
\mathcal{L}^{\prime}\left(\phi^{\prime}, \partial_{\mu} \phi^{\prime}\right)=\mathcal{L}\left(F\left(\phi^{\prime}\right), \partial_{\mu} F\left(\phi^{\prime}\right)\right)
$$

defines in general a new theory. Nevertheless, provided the transformation (2.4) has a Jacobian determinant equal to one at the origin, the S-matrix elements of the two theories are the same.

\footnotetext{
${ }^{1}$ compact, connected and semisimple
} 
From now on, we will refer to such transformations as allowed ones. The above theorem also holds order by order in perturbation theory.

Another useful result is that the more general non-linear realization of the group $G$ can be regarded as linear, once specialized to the subgroup $H$ of $G$.

- Theorem: If $H$ is the subgroup of $G$ leaving the origin invariant, then it is always possible to choose coordinates on $M$ so that:

$$
h y=D(h) y \quad \forall h \in H
$$

where $D(h)$ is a linear representation of $H$.

We sketch below the proof of this theorem. By expanding the element $h x$ in powers of $x$ around the origin one has:

$$
h x=D(h) x+O\left(x^{2}\right)
$$

In eq. (2.7), $O\left(x^{2}\right)$ denotes terms which are at least quadratic in $x$. The constant term is absent, due to the fact that $H$ leaves the origin invariant. We consider an invariant measure $d h$ on the group $H$, normalized so that:

$$
\int_{H} d h=1
$$

and we define the following coordinates on $M$ :

$$
\begin{aligned}
y & =\int_{H} d h D^{-1}(h) h x \\
& =x+O\left(x^{2}\right)
\end{aligned}
$$

Acting with an element $h_{0}$ of $H$ on the point $y$, one obtains:

$$
\begin{aligned}
h_{0} y & =\int_{H} d h D^{-1}(h) h h_{0} x \\
& =\int_{H} d\left(h h_{0}\right) D^{-1}\left(h h_{0} h_{0}^{-1}\right)\left(h h_{0}\right) x \\
& =D\left(h_{0}\right) y
\end{aligned}
$$

Notice that the transformation given in eq. (2.9) is an allowed one.

We now give an example of a non-linear realization of $G$ becoming linear when it is restricted to the subgroup $H$. As we shall see, this example plays a central role in our discussion and the non-linear realization dealt with is said to be in the standard form. To start with, one introduces a complete set of generators of $G$, 
$\left(V_{i}, A_{l}\right)$, orthonormal with respect to the inner Cartan product and such that the $V$ 's are generators of the subgroup $H$. Each element $g_{0}$ of $G$ admits the unique decomposition:

$$
g_{0}=e^{\xi A_{e} u V}
$$

where $\xi A=\xi_{l} A_{l}$ and $u V=u_{i} V_{i}$. For every element $g \in G$, one has:

$$
g e^{\xi A}=e^{\xi^{g} A} e^{u^{g} V}
$$

with

$$
\left\{\begin{array}{l}
\xi^{g}=\xi^{g}(\xi) \\
u^{g}=u^{g}(\xi)
\end{array}\right.
$$

The idea is to use $\xi$, the parameters related to the $A$ generators, as a subset of coordinates of $M$, with a transformation law under $G$ defined by eq. (2.13). To complete the set of coordinates, one introduces a vector $\psi$ carrying a linear representation of $H$ :

$$
\psi \rightarrow D(h) \psi \quad h \in H
$$

It is not difficult to show that the transformations

$$
\left\{\begin{array}{ccc}
\xi & \rightarrow & \xi^{g}(\xi) \\
\psi & \rightarrow & D\left(e^{u^{g}(\xi) V}\right) \psi
\end{array}\right.
$$

provide a non-linear realization of the group $G$, the so-called standard one. By restricting the transformations to $H$, one has:

$$
h e^{\xi A}=h e^{\xi A_{h}-1} h
$$

from which one deduces:

$$
\left\{\begin{array}{ccc}
e^{\xi A} & \rightarrow & h e^{\xi A_{h^{-1}}} \\
\psi & \rightarrow & D(h) \psi
\end{array}\right.
$$

Therefore, the transformations of $H$ on the standard coordinates are linearly realized.

The main result, not proved here, is contained in the following statement.

- Theorem: any non-linear realization of $\mathrm{G}$ can be put into the standard form by an allowed coordinate transformation.

This theorem solves the problem of characterizing all possible non-linear realization of $G$ on $M$. Its physical content is that, in discussing a theory describing the spontaneous breaking of the Lie group $G$ into a subgroup $H$, it is not restrictive 
to choose a set of fields transforming according to the standard form given in eq. (2.15).

- Example: we choose $G=S U(2)_{L} \otimes S U(2)_{R}$ and $H$ its diagonal subgroup, $S U(2)_{L+R}$. The generators of $G, L_{i}$ and $R_{i}(i=1,2,3)$, satisfy the following algebra:

$$
\begin{aligned}
{\left[L_{i}, L_{j}\right] } & =i \epsilon_{i j k} L_{k} \\
{\left[R_{i}, R_{j}\right] } & =i \epsilon_{i j k} R_{k} \\
{\left[L_{i}, R_{j}\right] } & =0
\end{aligned}
$$

As a realization of this algebra, one can take the following $4 \times 4$ matrices:

$$
L_{i}=\left(\begin{array}{cc}
\frac{\tau^{i}}{2} & 0 \\
0 & 0
\end{array}\right) \quad R_{i}=\left(\begin{array}{cc}
0 & 0 \\
0 & \frac{\tau^{i}}{2}
\end{array}\right)
$$

where $\tau^{i}$ are the Pauli matrices. The generators $(V, A)$ are given by:

$$
\begin{aligned}
V_{i} & =L_{i}+R_{i} \\
A_{i} & =L_{i}-R_{i}
\end{aligned}
$$

and satisfy the algebra:

$$
\begin{aligned}
{\left[V_{i}, V_{j}\right] } & =i \epsilon_{i j k} V_{k} \\
{\left[V_{i}, A_{j}\right] } & =i \epsilon_{i j k} A_{k} \\
{\left[A_{i}, A_{j}\right] } & =i \epsilon_{i j k} V_{k}
\end{aligned}
$$

To every generator $A_{i}$ we associate a coordinate $\xi_{i}$, with the transformation law given by:

$$
\begin{aligned}
g e^{i \xi A} & =e^{i \alpha A} e^{i \beta V} e^{i \xi A} \\
& =e^{i \xi^{g} A} e^{i u^{g} V}
\end{aligned}
$$

On the other hand, the group $G$ possesses an automorphism $g \rightarrow R(g)$, such that:

$$
\begin{aligned}
& V_{i} \rightarrow V_{i} \\
& A_{i} \rightarrow-A_{i}
\end{aligned}
$$

and we can write:

$$
R(g) e^{-i \xi A}=e^{-i \xi^{g} A} e^{i u^{g} V}
$$

By combining eqs. (2.22) and (2.24), one finally obtains:

$$
e^{2 i \xi^{g} A}=g e^{2 i \xi A} R\left(g^{-1}\right)
$$


or, more explicitly:

$$
e^{2 i \xi^{g} A}=e^{i \alpha A} e^{i \beta V} e^{2 i \xi A} e^{-i \beta V} e^{i \alpha A}
$$

From the last equality we can immediately see that, by specifying the transformations to the subgroup $H$, that is by putting the parameters $\alpha$ to zero, one has linear transformations for the coordinates $\xi$.

At this point we have to face the problem of constructing an invariant formalism out of the building block defined in eq. (2.15), the set of standard coordinates and their transformation properties. After promoting the coordinates on the manifold $M$ to scalar fields depending on the space-time points, we realize that, even in the case of global symmetry, the transformation laws given in eq. (2.15) are local, because of the explicit dependence on the fields $\xi$ 's. In order to work with objects depending on the derivatives of $\xi$ and $\psi$, with simple transformation properties under the group $G$, one can define appropriate covariant derivatives [33], dealing directly with the nonlinearly transforming fields $\xi$ and $\psi$. However, there is another possibility consisting in building functions of the fields $(\xi, \psi)$ which transform linearly under the group $G$. By combining such functions with the usual rules of representation theory, it is then straightforward to define invariant lagrangians. We will now show how to implement such a procedure.

Consider a linear representation $\mathcal{D}(g)$ of $G$ containing in its decomposition the representation $D(h)$ (see eqs. (2.14) and (2.15)). We define:

$$
\Phi=\mathcal{D}\left(e^{\xi A}\right) \psi
$$

The combination $\Phi$ given above transforms linearly under $G$, according to the representation $\mathcal{D}(g)$ :

$$
\begin{aligned}
\Phi^{\prime} & =\mathcal{D}\left(e^{\xi^{g} A}\right) D\left(e^{u^{g} V}\right) \psi \\
& =\mathcal{D}\left(g e^{\xi A} e^{-u^{g} V}\right) D\left(e^{u^{g} V}\right) \psi \\
& =\mathcal{D}(g) \mathcal{D}\left(e^{\xi A}\right) \psi \\
& =\mathcal{D}(g) \Phi
\end{aligned}
$$

- Example: we take $G=S U(2)_{L} \otimes S U(2)_{R}$ and $H=S U(2)_{L+R}$. Let $\sigma$ be an $S U(2)_{L+R}$-singlet, embedded into an $S U(2)_{L} \otimes S U(2)_{R}$ bidoublet:

$$
\psi=\left(\begin{array}{cc}
\sigma & 0 \\
0 & \sigma
\end{array}\right) \text {. }
$$


The action of the representation $\mathcal{D}(g)$ on a bidoublet $\Omega$ is defined as follows:

$$
\begin{aligned}
\Omega^{\prime} & =\mathcal{D}(g) \Omega \\
& =e^{i \vec{\lambda} \cdot \frac{\vec{\tau}}{2} \Omega e^{-i \vec{\rho} \cdot \frac{\vec{\tau}}{2}}}
\end{aligned}
$$

where $\vec{\lambda}$ and $\vec{\rho}$ are the parameters of the $S U(2)_{L} \otimes S U(2)_{R}$ transformations. It is easy to verify that $\psi$ is a singlet under the subgroup $S U(2)_{L+R}$, characterized by $\vec{\lambda}=\vec{\rho}$. According to eq. (2.27), we now define:

$$
\begin{aligned}
\Phi & =\mathcal{D}\left(e^{i \xi A}\right) \psi \\
& =e^{i \vec{\xi} \cdot \frac{\vec{\tau}}{2}}\left(\begin{array}{ll}
\sigma & 0 \\
0 & \sigma
\end{array}\right) e^{i \vec{\xi} \cdot \frac{\vec{\tau}}{2}} \\
& =\sigma e^{i \vec{\xi} \cdot \vec{\tau}}
\end{aligned}
$$

By construction, the function $\Phi=\Phi(\xi, \sigma)$ transforms as the bidoublet $\Omega$ of eq. (2.30). Explicitly we have:

$$
\begin{aligned}
\sigma^{\prime} & =\sigma \\
e^{i \overrightarrow{\xi^{\prime}} \cdot \vec{\tau}} & =e^{i \vec{\lambda} \cdot \frac{\vec{\tau}}{2}} e^{i \vec{\xi} \cdot \vec{\tau}} e^{-i \vec{\rho} \cdot \frac{\vec{\tau}}{2}}
\end{aligned}
$$

To take into account the dimension of the fields $\xi$, one usually performs the following rescaling:

$$
\xi_{i} \rightarrow \frac{\xi_{i}}{f}
$$

where $f$ is a constant with the dimension of a mass. A lagrangian invariant under global transformations of $S U(2)_{L} \otimes S U(2)_{R}$ is:

$$
\begin{aligned}
\mathcal{L} & =\frac{f^{2}}{4} \operatorname{tr}\left(\partial_{\mu} U^{\dagger} \partial^{\mu} U\right) \\
& =\frac{1}{2} \partial_{\mu} \xi_{i} \partial^{\mu} \xi_{i}+\ldots
\end{aligned}
$$

where:

$$
U=e^{i \frac{\vec{\xi} \cdot \vec{\tau}}{f}}
$$

This is the lagrangian of the well-known non-linear $\sigma$-model [1]. The global invariance under $S U(2)_{L} \otimes S U(2)_{R}$ is spontaneously broken down to $S U(2)_{L+R}$. The fields $\xi_{i}$, associated to the broken generators $A_{i}$, are the Goldstone bosons. The dots in eq. (2.35) denote higher order terms in the Goldstone fields. 
- Exercise: verify that the transformation law for $U$ (see eq.(2.33)), agrees with the transformation law for $\xi$ given in eq. (2.26).

- Exercise: build the gauged non-linear $\sigma$-model by gauging the entire group $G=$ $S U(2)_{L} \otimes S U(2)_{R}$. By going to the unitary gauge, discuss the physical degrees of freedom and their mass spectrum. 


\section{Electroweak Interactions: the Lowest Order}

In this section we review the construction of the standard model of electroweak interactions and of some of its variants, by explicitly applying the formalism developed in the previous lecture. These models are characterized by an invariance under the gauge group $G=S U(2)_{L} \otimes U(1)_{Y}$, spontaneously broken down to the local subgroup $H=U(1)_{e m}$. The generators $T^{i}(i=1,2,3)$ and $Y$ of $S U(2)_{L} \otimes U(1)_{Y}$ satisfy the following algebra:

$$
\begin{aligned}
{\left[T_{i}, T_{j}\right] } & =i \epsilon_{i j k} T_{k} \\
{\left[T_{i}, Y\right] } & =0 \\
{[Y, Y] } & =0
\end{aligned}
$$

The generators of the subgroup $U(1)_{e m}$ and of the coset $S U(2)_{L} \otimes U(1)_{Y} / U(1)_{e m}$ are given by 27:

$$
\begin{aligned}
X_{e m} & =\left(T^{3}+Y\right) \longleftrightarrow U(1)_{e m} \\
Y^{i} & =T^{i} \longleftrightarrow S U(2)_{L} \otimes U(1)_{Y} / U(1)_{e m}
\end{aligned}
$$

We begin by introducing the would-be Goldstone bosons and the Higgs field. We consider a singlet $\sigma$ under $U(1)_{e m}$, embedded into a doublet representation $\mathcal{D}$, of hypercharge $Y=1 / 2$ :

$$
\varphi=\left(\begin{array}{l}
0 \\
\sigma
\end{array}\right)
$$

The action of the representation $\mathcal{D}(g)$ on a doublet $\chi$ is defined below:

$$
\begin{aligned}
\chi^{\prime} & =\mathcal{D}(g) \chi \\
& =e^{i \vec{\alpha} \cdot \frac{\vec{\tau}}{2} e^{\alpha_{Y}} \frac{1}{2} \chi}
\end{aligned}
$$

In the equation above, $\alpha_{i}$ and $\alpha_{Y}$ are the local parameters of the $S U(2)_{L}$ and $U(1)_{Y}$ gauge transformations, respectively. It is immediate to verify that $\varphi$ is a singlet under the unbroken group $U(1)_{\text {em }}$. Following the prescription given in eq. (2.27), we define:

$$
\begin{aligned}
\Phi & =\mathcal{D}\left(e^{i \vec{\xi} \cdot \vec{Y}}\right) \varphi \\
& =e^{i \vec{\xi} \cdot \frac{\tau}{2}}\left(\begin{array}{l}
0 \\
\sigma
\end{array}\right)
\end{aligned}
$$

\footnotetext{
${ }^{2}$ different choices for the generators of the coset are also possible
} 
By construction $\Phi$ transforms as a doublet with hypercharge $Y=1 / 2$. We prefer to work with a linear multiplet written in a matrix form and, to this purpose, we introduce the new doublet:

$$
\begin{aligned}
\tilde{\Phi} & =i \tau^{2} \Phi^{*} \\
& =e^{i \vec{\xi} \cdot \frac{\vec{\tau}}{2}}\left(\begin{array}{c}
\sigma \\
0
\end{array}\right)
\end{aligned}
$$

The doublet $\tilde{\Phi}$ has hypercharge $Y=-1 / 2$. We define the $2 \times 2$ matrix $M$ :

$$
\begin{aligned}
M & =(\tilde{\Phi} \Phi) \\
& =\sigma e^{i \vec{\xi} \cdot \frac{\vec{\tau}}{2}}
\end{aligned}
$$

The matrix $M$ transforms as follows:

$$
M^{\prime}=g_{L} M g_{R}^{\dagger}
$$

where:

$$
\begin{aligned}
g_{L} & =e^{i \vec{\alpha} \cdot \frac{\vec{\tau}}{2}} \\
g_{R} & =e^{i \alpha_{Y} \frac{\tau^{3}}{2}}
\end{aligned}
$$

Explicitly, one has:

$$
\begin{aligned}
\sigma^{\prime} & =\sigma \\
e^{i \overrightarrow{\xi^{\prime}} \cdot \frac{\vec{\tau}}{2}} & =g_{L} e^{i \vec{\xi} \cdot \frac{\vec{\tau}}{2} g_{R}^{\dagger}}
\end{aligned}
$$

The Higgs field, $\sigma$, is invariant under the whole gauge group $S U(2)_{L} \otimes U(1)_{Y}$. The would-be Goldstone bosons transform non-linearly under $S U(2)_{L} \otimes U(1)_{Y}$. However, the combination:

$$
U=e^{i \frac{\vec{\xi} \cdot \vec{\tau}}{v}}
$$

transforms linearly as specified in eq. (3.12). In the previous equality we have rescaled the fields $\xi$, introducing the constant $v$. Let us forget for a while the Higgs field $\sigma$ and proceed to define the lagrangian for the bosonic fields. The covariant derivative for the combination $U$ is defined as follows:

$$
D_{\mu} U=\partial_{\mu} U-g \hat{W}_{\mu} U+g^{\prime} U \hat{B}_{\mu}
$$


where $g$ and $g^{\prime}$ are the gauge coupling constants for $S U(2)_{L}$ and $U(1)_{Y} ; \hat{W}_{\mu}, \hat{B}_{\mu}$ are the gauge fields, written as matrices:

$$
\begin{aligned}
\hat{W}_{\mu} & =\frac{1}{2 i} \vec{W}_{\mu} \cdot \vec{\tau} \\
\hat{B}_{\mu} & =\frac{1}{2 i} B_{\mu} \tau^{3}
\end{aligned}
$$

The corresponding field strengths are given by:

$$
\begin{aligned}
\hat{W}_{\mu \nu} & =\partial_{\mu} \hat{W}_{\nu}-\partial_{\nu} \hat{W}_{\mu}-g\left[\hat{W}_{\mu}, \hat{W}_{\nu}\right] \\
\hat{B}_{\mu \nu} & =\partial_{\mu} \hat{B}_{\nu}-\partial_{\nu} \hat{B}_{\mu}
\end{aligned}
$$

Their transformation laws are the following:

$$
\begin{aligned}
\hat{W}_{\mu}^{\prime} & =g_{L} \hat{W}_{\mu} g_{L}^{\dagger}-\frac{1}{g} g_{L} \partial_{\mu} g_{L}^{\dagger} \\
\hat{B}_{\mu}^{\prime} & =\hat{B}_{\mu}-\frac{1}{g^{\prime}} g_{R} \partial_{\mu} g_{R}^{\dagger}
\end{aligned}
$$

and

$$
\begin{aligned}
\hat{W}_{\mu \nu}^{\prime} & =g_{L} \hat{W}_{\mu \nu} g_{L}^{\dagger} \\
\hat{B}_{\mu \nu}^{\prime} & =\hat{B}_{\mu \nu}
\end{aligned}
$$

The lagrangian for the bosonic fields is given by:

$$
\mathcal{L}_{B}=\frac{1}{2} \operatorname{tr}\left(\hat{W}_{\mu \nu} \hat{W}^{\mu \nu}+\hat{B}_{\mu \nu} \hat{B}^{\mu \nu}\right)+\frac{v^{2}}{4} \operatorname{tr}\left(D_{\mu} U^{\dagger} D^{\mu} U\right)
$$

From the last term in the previous equation, by going to the unitary gauge $U=$ $\mathbf{1}$, we can read the mass term for the gauge vector bosons. One introduces the combinations:

$$
\begin{aligned}
W_{\mu}^{ \pm} & =\frac{\left(W_{\mu}^{1} \mp i W_{\mu}^{2}\right)}{\sqrt{2}} \\
Z_{\mu} & =\cos \theta W_{\mu}^{3}-\sin \theta B_{\mu} \\
A_{\mu} & =\sin \theta W_{\mu}^{3}+\cos \theta B_{\mu}
\end{aligned}
$$

where the angle $\theta$ is defined by:

$$
\tan \theta=\frac{g^{\prime}}{g}
$$

The mass spectrum in the gauge vector boson sector is given in table I. 


\begin{tabular}{|c|c|}
\hline & $(\text { mass })^{2}$ \\
\hline$W^{ \pm}$ & $\frac{v^{2} g^{2}}{4}$ \\
\hline$Z$ & $\frac{v^{2}\left(g^{2}+g^{\prime 2}\right)}{4}$ \\
\hline$A$ & 0 \\
\hline
\end{tabular}

Table I: electroweak gauge vector boson masses.

Now we consider the lagrangian $\mathcal{L}_{\psi}$ for the fermionic fields, whose quantum numbers are listed in table II.

\begin{tabular}{|c|c|c|c|}
\hline$\psi$ & $S U(2)_{L}$ & $Y$ & $\frac{(B-L)}{2}$ \\
\hline$q_{L}=\left(\begin{array}{c}u_{L} \\
d_{L}\end{array}\right)$ & $\underline{2}$ & $1 / 6$ & $1 / 6$ \\
\hline$q_{R}=\left(\begin{array}{c}u_{R} \\
d_{R}\end{array}\right)$ & $\underline{1}$ & $\begin{array}{c}2 / 3 \\
-1 / 3\end{array}$ & $\begin{array}{c}1 / 6 \\
1 / 6\end{array}$ \\
\hline$l_{L}=\left(\begin{array}{c}\nu_{L} \\
e_{L}\end{array}\right)$ & $\underline{2}$ & $-1 / 2$ & $-1 / 2$ \\
\hline$l_{R}=\left(\begin{array}{c}0 \\
e_{R}\end{array}\right)$ & $\underline{1}$ & -1 & $-1 / 2$ \\
\hline
\end{tabular}

Table II: fermions and their electroweak quantum numbers.

The kinetic terms for the fermionic fields and their minimal coupling to the gauge vector bosons are given by:

$$
\begin{aligned}
\mathcal{L}_{\psi} & =i \bar{q}_{L} \gamma^{\mu} D_{\mu} q_{L}+i \bar{q}_{R} \gamma^{\mu} D_{\mu} q_{R}+ \\
& +i \bar{l}_{L} \gamma^{\mu} D_{\mu} l_{L}+i \bar{l}_{R} \gamma^{\mu} D_{\mu} l_{R}
\end{aligned}
$$

Indices in the generation space are understood here. The covariant derivatives acting on the left and right-handed fermions $\psi_{L, R},(\psi=q, l)$ are defined below:

$$
\begin{aligned}
D_{\mu} \psi_{L} & =\left(\partial_{\mu}-g \hat{W}_{\mu}-g^{\prime} \hat{B}_{\mu}^{(L)}\right) \psi_{L} \\
D_{\mu} \psi_{R} & =\left(\partial_{\mu}-g^{\prime} \hat{B}_{\mu}^{(R)}\right) \psi_{R}
\end{aligned}
$$

The combinations $\hat{B}_{\mu}^{(L, R)}$ are given by:

$$
\begin{aligned}
\hat{B}_{\mu}^{(L)} & =\frac{1}{2 i}(B-L) B_{\mu} \\
\hat{B}_{\mu}^{(R)} & =\frac{1}{2 i}\left(\tau^{3}+(B-L)\right) B_{\mu}
\end{aligned}
$$


As a function of the mass eigenstates for the gauge vector bosons (eq. (3.20)), the lagrangian given in eq. (3.22) contains the following interaction terms:

$$
-\frac{g}{\sqrt{2}}\left(W_{\mu}^{+} J^{+^{\mu}}+W_{\mu}^{-} J^{-\mu}\right)-e A_{\mu} J_{e m}^{\mu}-\frac{g}{\cos \theta} Z_{\mu} J_{Z}^{\mu}
$$

where

$$
\begin{aligned}
J^{ \pm \mu} & =\sum_{\psi} \bar{\psi}_{L} \gamma^{\mu} \tau^{ \pm} \psi_{L} \\
J_{e m}{ }^{\mu} & =\sum_{\psi} Q_{e m}^{\psi} \bar{\psi} \gamma^{\mu} \psi \\
J_{Z}{ }^{\mu} & =\left(J_{3 L}^{\mu}-\sin ^{2} \theta J_{e m}^{\mu}\right) \\
J_{3 L}{ }^{\mu} & =\sum_{\psi} \bar{\psi}_{L} \gamma^{\mu} \frac{\tau^{3}}{2} \psi_{L}
\end{aligned}
$$

As usual, one has $\tau^{ \pm}=\left(\tau^{1} \pm i \tau^{2}\right) / 2, Q_{e m}=T^{3}+Y$ and $e=g \sin \theta$.

The mass terms for quarks and leptons are introduced by means of gauge invariant Yukawa interaction terms, $\mathcal{L}_{Y}$. We have:

$$
\mathcal{L}_{Y}=\bar{q}_{L} U m_{q} q_{R}+\bar{l}_{L} U m_{l} l_{R}+\text { h.c. }
$$

$\mathcal{L}_{Y}$ is invariant under $S U(2)_{L} \otimes U(1)_{Y}$ provided the mass matrices $m_{q}$ and $m_{l}$ are linear combinations of $\mathbf{1}$ and $\tau^{3}$, in the corresponding two-dimensional flavour space. This means that one has independent mass matrices for each separate charge $u, d, e$.

By ignoring the Higgs degree of freedom, the total lagrangian is given by:

$$
\mathcal{L}=\mathcal{L}_{B}+\mathcal{L}_{\psi}+\mathcal{L}_{Y}
$$

This model can be easily extended to account for the presence of the Higgs particle, here identified with a real scalar field $\sigma$, invariant under the whole gauge group $S U(2)_{L} \otimes U(1)_{Y}$ (see eq. (3.11)). One has simply to add to the lagrangian $\mathcal{L}$, additional terms of the form:

$$
\begin{aligned}
\mathcal{L}_{\sigma} & =\frac{1}{2} \partial_{\mu} \sigma \partial^{\mu} \sigma-V(\sigma)+ \\
& +\left[a\left(\frac{\sigma}{v}\right) \frac{v^{2}}{4} \operatorname{tr}\left(D_{\mu} U^{\dagger} D^{\mu} U\right)+b\left(\frac{\sigma}{v}\right)^{2} \frac{v^{2}}{4} \operatorname{tr}\left(D_{\mu} U^{\dagger} D^{\mu} U\right)+\ldots\right] \\
& +\left[\left(\frac{\sigma}{v}\right) \bar{q}_{L} U M_{q} q_{R}+\left(\frac{\sigma}{v}\right) \bar{l}_{L} U M_{l} l_{R}+\text { h.c. }\right]
\end{aligned}
$$

Since $\sigma$ is a singlet under the entire gauge group, other interactions could be easily added to the lagrangian $\mathcal{L}_{\sigma}$, which contains just few possible terms. Notice that 
$a, b, \ldots$ are arbitrary real parameters, and $M_{q, l}$ are matrices, linear combinations of 1 and $\tau^{3}$, not necessarily equal to the matrices $m_{q, l} . V(\sigma)$ denotes here the scalar potential for the Higgs field.

The usual standard model of the electroweak interactions is a specialization of the lagrangian $\mathcal{L}+\mathcal{L}_{\sigma}$ to the following choice of $a, b, M_{q}, M_{l}$ and $V$ :

$$
\begin{aligned}
a & =2 \\
b & =1 \\
M_{q} & =m_{q} \\
M_{l} & =m_{l} \\
V\left((\sigma+v)^{2}\right) & =\frac{m_{\sigma}^{2}}{8 v^{2}}\left((\sigma+v)^{2}-v^{2}\right)^{2}
\end{aligned}
$$

With the above choice, we can easily rewrite the lagrangian $\mathcal{L}+\mathcal{L}_{\sigma}$ in the form:

$$
\begin{aligned}
\mathcal{L}_{S M} & =\frac{1}{2} \operatorname{tr}\left(\hat{W}_{\mu \nu} \hat{W}^{\mu \nu}+\hat{B}_{\mu \nu} \hat{B}^{\mu \nu}\right)+\frac{1}{4} \operatorname{tr}\left(D_{\mu} M^{\dagger} D^{\mu} M\right)-V\left(M^{\dagger} M\right)+ \\
& +\mathcal{L}_{\psi}+\left[\bar{q}_{L} M \frac{m_{q}}{v} q_{R}+\bar{l}_{L} M \frac{m_{l}}{v} l_{R}+\text { h.c. }\right]
\end{aligned}
$$

where:

$$
M=\sigma U=\sigma e^{i \frac{\vec{\xi} \cdot \vec{\tau}}{v}}
$$

Finally, to obtain the lagrangian for the standard model in a more familiar form, we can perform the following field redefinition:

$$
M=\sqrt{2}\left(\begin{array}{cc}
\varphi^{0} & -\varphi^{+} \\
\varphi^{-} & \left(\varphi^{0}\right)^{*}
\end{array}\right)
$$

Taking into account the vacuum expectation value $v$ of the field $\sigma$ in eq. (3.37), we realize that this field transformation is an allowed one and the S-matrix elements of the theory remain unchanged. We recognize in

$$
\left(\begin{array}{c}
\varphi^{0} \\
\varphi^{-}
\end{array}\right)
$$

the usual doublet of scalar fields.

Up to now, Higgs particles have not been detected, and on the standard Higgs mass, the LEP collaborations have put a lower bound of $60 \mathrm{GeV}$ 36. From this 
point of view, there is no reason to prefer, as an effective model for low-energy electroweak interactions, one particular model among $\left.\mathcal{L}, \mathcal{L}+\mathcal{L}_{\sigma}\right\}, \mathcal{L}_{S M}$. However, an important property is enjoyed only by $\mathcal{L}_{S M}$ : the renormalizability. With the increasing accumulation of precision tests of the electroweak theory, the compelling need to take into account quantum effects in comparing predictions and measurements makes mandatory the use of a consistent framework for the evaluation of the radiative corrections, that is a renormalizable theory. Nevertheless, as shown in the following sections, there is an interesting use one can make of non-renormalizable, effective lagrangians, in connection with the existence of something beyond the standard model.

We conclude this section with a remark. The lagrangian $\mathcal{L}+\mathcal{L}_{\sigma}$ is not the most general lagrangian invariant under $S U(2)_{L} \otimes U(1)_{Y}$, containing up to two derivatives. By taking advantage of the formalism of non-linear realizations, one could introduce interactions between fermions and gauge bosons which explicitly violate the minimal form given in eq. (3.22) 37]. As an example, we consider a left-handed quark $b_{L}$ of electric charge $-1 / 3$, embedded in a $S U(2)_{L}$ doublet with hypercharge $Y=1 / 6$ :

$$
\left(\begin{array}{c}
0 \\
b_{L}
\end{array}\right)
$$

The corresponding linear multiplet, defined according the eq. (2.27), is given by:

$$
\Psi=U\left(\begin{array}{c}
0 \\
b_{L}
\end{array}\right)
$$

It is then immediate to verify that the interaction term

$$
\operatorname{tr}\left(\tau^{3} U^{\dagger} D_{\mu} U\right) \overline{b_{L}} \gamma^{\mu} b_{L}
$$

is gauge invariant and provides a modification of the tree-level standard model $Z b \bar{b}$ coupling. Non-minimal terms of this kind arise for instance in the low energy limit of the standard model for a large top mass [17].

\footnotetext{
${ }^{3}$ Bounds on the matrices $M_{q, l}$ in $\mathcal{L}_{\sigma}$ come from data on flavour changing processes
} 


\section{Precision Measurements and Electroweak Ra- diative corrections}

At the moment there is a quite astonishing agreement between the predictions of the standard model and the whole set of data from precision measurements. The LEP data have certainly played a major role in testing with great accuracy the standard model theory. The agreement has been pushed to the level of checking the radiative corrections, namely the predictions of the model including the most relevant quantum effects.

Associated to the problem of computing the radiative corrections, there is an obstacle given by the presence of infinities in the intermediate steps of the computation. Such infinities are dealt with by a renormalization procedure. In practice, starting from a lagrangian $\mathcal{L}(g)$ depending on a set of coupling constants $g$, one introduces a regulator $\Lambda$ to give a mathematical meaning to the expressions obtained. The physical amplitudes computed in the regulated theory $\mathcal{L}(g, \Lambda)$, diverge in the limit of infinite $\Lambda$. Order by order in perturbation theory, a suitable set of counterterms $\sum_{i} \delta g_{i} C_{i}$ is added to the original lagrangian $\mathcal{L}(g, \Lambda)$, so that each amplitude is finite, at that given order. The ambiguities related to the introduction of divergent counterterms are removed by the requirement of specific renormalization conditions, defining the renormalized parameters of the theory.

The basic property of renormalizable theories is that the counterterms can be absorbed by redefining the parameters of the original theory:

$$
g \rightarrow g_{0}=g+\delta g
$$

In this way, at all orders in perturbation theory, the predictions of the theory depend on a given number of parameters, which can be determined by a finite number of independent measurements. In theories characterized by global or local symmetries, a great simplification of the renormalization procedure is obtained by adopting a regularization preserving the symmetries. However, there are cases in which classical symmetries are violated at the quantum level [20].

In the following, we will assume that we have computed, for a particular model, some radiative corrections and we will discuss how the physical quantities are affected by them. To simplify the analysis, we will make the following hypotheses [38, 6, 7, 39, 40]:

(i) The radiative effects are related to a mass scale $M$ much greater than the electroweak scale $M_{Z}$. 
(ii) The radiative effects are dominated by the gauge bosons self-energy corrections, at least for a suitable set of measurable quantities 1 .

These assumptions are both violated in the standard model 41]. However, they can be fulfilled in some of its extensions, at least for that part of quantum effects having a non-standard origin. Later on we will provide some examples.

We will denote by $-i \Pi_{i j}^{\mu \nu}(p)$ the set of self-energy corrections for the gauge boson fields, evaluated at some loop order 9 . The indices $i, j$ can take the values 0 (for the field $B$ ) and 1,2,3 (for the fields $W^{i}$ ), or, alternatively, the values $\gamma, Z, W$. One has:

$$
-i \Pi_{i j}^{\mu \nu}(p)=-i\left[\Pi_{i j}\left(p^{2}\right) g^{\mu \nu}+\left(p^{\mu} p^{\nu} \text { terms }\right)\right]
$$

The terms proportional to $p^{\mu} p^{\nu}$ have no practical effect for the LEP I physics, and they will be disregarded from now on. The scalar function $\Pi_{i j}\left(p^{2}\right)$ can be expanded around the point $p^{2}=0$ :

$$
\Pi_{i j}\left(p^{2}\right)=A_{i j}+p^{2} F_{i j}+\ldots
$$

According to our assumption (i), this expansion, meaningful for $p^{2} \ll M^{2}$, will contain real coefficients $A_{i j}, F_{i j}$, etc. Moreover, since $\Pi_{i j}\left(p^{2}\right)$ has dimension two in units of mass, it will be reasonable to neglect the dots in eq. (4.3), representing terms suppressed by positive powers of $\left(p^{2} / M^{2}\right)$.

As a consequence of the exact electromagnetism gauge invariance, we have $A_{\gamma \gamma}=$ $A_{\gamma Z}=0$. Then we are left with the six independent coefficients $A_{Z Z}, A_{W W}, F_{\gamma \gamma}$, $F_{\gamma Z}, F_{Z Z}, F_{W W}$. The measurable quantities will depend on these six parameters. However, three combinations of them are related to very special observables, which, in the electroweak theory, play the role of fundamental constants. These are given by the electromagnetic fine structure constant $\alpha$, the Fermi constant $G_{F}$ and the mass of the $Z$ gauge vector boson $M_{Z}$. It is immediate to verify that the shifts in the fundamental constants, due to the quantum corrections induced by the gauge vector boson self-energies, are given by:

$$
\begin{aligned}
\frac{\delta \alpha}{\alpha} & =-F_{\gamma \gamma} \\
\frac{\delta G_{F}}{G_{F}} & =\frac{A_{W W}}{M_{W}^{2}} \\
\frac{\delta M_{Z}^{2}}{M_{Z}^{2}} & =-\left(\frac{A_{Z Z}}{M_{Z}^{2}}+F_{Z Z}\right)
\end{aligned}
$$

\footnotetext{
${ }^{4}$ In the following we will be mostly concerned with the LEP I physics.

${ }^{5}$ We are following here the presentation given in ref. 39]
} 
For future reference, we also give the shift for the $W$ vector boson mass:

$$
\frac{\delta M_{W}^{2}}{M_{W}^{2}}=-\left(\frac{A_{W W}}{M_{W}^{2}}+F_{W W}\right)
$$

- Exercise: derive eqs. (4.4-4.7).

We conclude that, in our approximation, the parameters counting independent measurable effects, induced at the quantum level by the underlying theory, are three combinations among the six coefficients $A_{Z Z}, A_{W W}, F_{\gamma \gamma}, F_{\gamma Z}, F_{Z Z}, F_{W W}$.

To identify these combinations, we will now compute the radiative corrections for the three following observables: the ratio of the gauge boson masses $M_{W} / M_{Z}$, the forward-backward asymmetry $A_{F B}^{\mu}$ in $e^{+} e^{-} \rightarrow \mu^{+} \mu^{-}$at the $Z$ peak and the partial width of the $Z$ into charged leptons, $\Gamma_{l}$.

- $\frac{M_{W}}{M_{Z}}$

We trade $M_{W} / M_{Z}$ for the observable $\Delta r_{W}$ defined as follows:

$$
\left(\frac{M_{W}}{M_{Z}}\right)^{2}=\frac{1}{2}+\sqrt{\frac{1}{4}-\frac{\mu^{2}}{M_{Z}^{2}\left(1-\Delta r_{W}\right)}}
$$

where:

$$
\mu^{2}=\frac{\pi \alpha\left(M_{Z}^{2}\right)}{\sqrt{2} G_{F}}=(38.454 G e V)^{2}
$$

The definition given in eqs. (4.8-4.9) is suggested by the lowest order relation:

$$
\left(\frac{m_{W}}{m_{Z}}\right)^{2}=\frac{1}{2}+\sqrt{\frac{1}{4}-\frac{\mu_{0}^{2}}{m_{Z}^{2}}}
$$

with:

$$
\mu_{0}^{2}=\frac{\pi \alpha_{0}}{\sqrt{2} G_{F}^{0}}
$$

and

$$
\begin{aligned}
\alpha & =\alpha_{0}\left(1+\frac{\delta \alpha}{\alpha}\right) \\
G_{F} & =G_{F}^{0}\left(1+\frac{\delta G_{F}}{G_{F}}\right) \\
M_{W, Z}^{2} & =m_{W, Z}^{2}\left(1+\frac{\delta M_{W, Z}^{2}}{M_{W, Z}^{2}}\right)
\end{aligned}
$$


By combining eqs. (4.8-4.14), one obtains:

$$
\left(\frac{M_{W}}{M_{Z}}\right)^{2}=\left(\frac{m_{W}}{m_{Z}}\right)^{2}\left(1-\frac{\sin ^{2} \theta}{\cos 2 \theta}\left(\Delta r_{W}-\frac{\delta M_{Z}^{2}}{M_{Z}^{2}}+\frac{\delta \alpha}{\alpha}-\frac{\delta G_{F}}{G_{F}}\right)\right)
$$

On the other hand, one has:

$$
\left(\frac{M_{W}}{M_{Z}}\right)^{2}=\left(\frac{m_{W}}{m_{Z}}\right)^{2}\left(1+\frac{\delta M_{W}^{2}}{M_{W}^{2}}-\frac{\delta M_{Z}^{2}}{M_{Z}^{2}}\right)
$$

By comparing eqs. (4.15) and (4.16) and by using eqs. (4.4-4.7), one finds:

$$
\begin{aligned}
\Delta r_{W} & =-\frac{\cos ^{2} \theta}{\sin ^{2} \theta}\left(\frac{A_{Z Z}}{M_{Z}^{2}}-\frac{A_{W W}}{M_{W}^{2}}\right)+ \\
& +\frac{\cos 2 \theta}{\sin ^{2} \theta}\left(F_{W W}-F_{33}\right)+ \\
& +2 \frac{\cos \theta}{\sin \theta} F_{30}
\end{aligned}
$$

- $\mathbf{A}_{\mathbf{F B}}^{\mu}$

Also in this case we proceed through a series of definitions inspired to the lowest order relations:

$$
\begin{gathered}
A_{F B}^{\mu}\left(p^{2}=M_{Z}^{2}\right)=3\left(\frac{g_{V} g_{A}}{g_{V}^{2}+g_{A}^{2}}\right)^{2} \\
g_{V}=-\frac{1}{2}+2 \sin ^{2} \hat{\theta} \\
g_{A}=\frac{1}{2} \\
\sin ^{2} \hat{\theta}=(1+\Delta k) \sin ^{2} \tilde{\theta} \\
\sin ^{2} \tilde{\theta}=\frac{1}{2}-\sqrt{\frac{1}{4}-\frac{\mu^{2}}{M_{Z}^{2}}} \\
=0.23145 \quad\left(\text { for } M_{Z}=91.175 \mathrm{GeV}\right)
\end{gathered}
$$

With these definitions, the knowledge of $A_{F B}^{\mu}$ is equivalent to that of the parameter $\Delta k$, given in eq. (4.21). To determine the latter, we focus on the neutral current scattering process $e^{+} e^{-} \rightarrow \mu^{+} \mu^{-}$, with electrons and muons in the right-handed 
polarization state. The lowest order amplitude to this process is derived by the interaction lagrangian (see eqs. (3.27-3.31)):

$$
\left(e_{0} A_{\mu}-g_{0} \frac{\sin ^{2} \theta_{0}}{\cos \theta_{0}} Z_{\mu}\right) J_{e m}^{\mu}
$$

and is proportional to:

$$
i\left[\frac{e_{0}^{2}}{p^{2}}+\left(-g_{0} \frac{\sin ^{2} \theta_{0}}{\cos \theta_{0}}\right)^{2} \frac{1}{\left(p^{2}-m_{Z}^{2}\right)}\right]
$$

The self-energy corrections induce the terms:

$$
\begin{aligned}
& -i e_{0}^{2} \frac{F_{\gamma \gamma}}{p^{2}}- \\
& -i g_{0}^{2} \frac{\sin ^{4} \theta_{0}}{\cos ^{2} \theta_{0}} \frac{1}{\left(p^{2}-m_{Z}^{2}\right)} \frac{A_{Z Z}+p^{2} F_{Z Z}}{\left(p^{2}-m_{Z}^{2}\right)}+ \\
& +2 i\left(e_{0} g_{0}\right) \frac{\sin ^{2} \theta_{0}}{\cos \theta_{0}} \frac{F_{\gamma Z}}{\left(p^{2}-m_{Z}^{2}\right)}
\end{aligned}
$$

The first term of eq. (4.24) combines with the first term in eq. (4.25), giving a shift of the constant $\alpha$, as given by eq. (4.4). The sum of the remaining terms, up to higher order corrections, is given by:

$$
i \frac{g_{0}^{2}}{\cos ^{2} \theta_{0}}\left(1-F_{Z Z}\right)\left(-\sin ^{2} \theta_{0}\right)^{2}\left(1+2 \frac{\cos \theta}{\sin \theta} F_{\gamma Z}\right) \frac{1}{\left(p^{2}-M_{Z}^{2}\right)}
$$

The factor $\left(1-F_{Z Z}\right)$ in the previous expression represents a universal correction for the neutral current. (It is the analogue of the factor $\left(1-F_{\gamma \gamma}\right)$ for the electromagnetic current.) To see this, one can consider the scattering process $\nu_{e} \nu_{e} \rightarrow \nu_{\mu} \nu_{\mu}$, whose amplitude is proportional to:

$$
i \frac{g_{0}^{2}}{\cos ^{2} \theta_{0}}\left(1-F_{Z Z}\right) \frac{1}{\left(p^{2}-M_{Z}^{2}\right)}
$$

In conclusion, the self-energy corrections can be accounted for by an effective neutral current lagrangian given by:

$$
\frac{g_{0}}{\cos \theta_{0}} \sqrt{1-F_{Z Z}}\left[J_{3 L}^{\mu}-\sin ^{2} \theta_{0}\left(1+\frac{\cos \theta}{\sin \theta} F_{\gamma Z}\right) J_{e m}^{\mu}\right] Z_{\mu}
$$

From this lagrangian, one can read the effective Weinberg angle $\sin ^{2} \hat{\theta}$ :

$$
\sin ^{2} \hat{\theta}=\left(1+\frac{\cos \theta}{\sin \theta} F_{\gamma Z}\right) \sin ^{2} \theta_{0}
$$


On the other hand, from the definition of $\sin ^{2} \tilde{\theta}$ given in eq. (4.21), one has:

$$
\sin ^{2} \tilde{\theta}=\sin ^{2} \theta_{0}\left(1+\frac{\cos ^{2} \theta}{\cos 2 \theta}\left(\frac{\delta \alpha}{\alpha}-\frac{\delta G_{F}}{G_{F}}-\frac{\delta M_{Z}^{2}}{M_{Z}^{2}}\right)\right)
$$

By comparing eqs. (4.29) and (4.30) and by making use of eqs. (4.4-4.7), one finds:

$$
\begin{aligned}
\Delta k= & -\frac{\cos ^{2} \theta}{\cos 2 \theta}\left(\frac{A_{Z Z}}{M_{Z}^{2}}-\frac{A_{W W}}{M_{W}^{2}}\right) \\
& +\frac{1}{\cos 2 \theta} \frac{\cos \theta}{\sin \theta} F_{30}
\end{aligned}
$$

- $\Gamma_{l}$

We define $\Gamma_{l}$ as follows:

$$
\Gamma_{l}=\frac{G_{F} M_{Z}^{3}}{6 \pi \sqrt{2}}(1+\Delta \rho)\left(g_{V}^{2}+g_{A}^{2}\right)
$$

To compute the parameter $\Delta \rho$, one can refer to the effective neutral current lagrangian of eq. (4.28). This contains the overall factor:

$$
\frac{g_{0}}{\cos \theta_{0}} \sqrt{1-F_{Z Z}}
$$

We relate this factor to the physical constants $G_{F}$ and $M_{Z}$. One has:

$$
4 \sqrt{2} G_{F} M_{Z}^{2}=\frac{g_{0}^{2}}{\cos ^{2} \theta_{0}}\left(1+\frac{\delta G_{F}}{G_{F}}+\frac{\delta M_{Z}^{2}}{M_{Z}^{2}}\right)
$$

From the previous equation, and from eqs. (4.4-4.7), one obtains:

$$
\frac{g_{0}^{2}}{\cos ^{2} \theta_{0}}\left(1-F_{Z Z}\right)=4 \sqrt{2} G_{F} M_{Z}^{2}\left(1+\left(\frac{A_{Z Z}}{M_{Z}^{2}}-\frac{A_{W W}}{M_{W}^{2}}\right)\right)
$$

Therefore, the parameter $\Delta \rho$ is given by:

$$
\Delta \rho=\frac{A_{Z Z}}{M_{Z}^{2}}-\frac{A_{W W}}{M_{W}^{2}}
$$

By looking at the expressions we have obtained for the quantities $\Delta r_{W}, \Delta k$ and $\Delta \rho$, we recognize that they depend on the following three combinations of self-energy 
corrections:

$$
\begin{aligned}
\epsilon_{1} & =\frac{A_{Z Z}}{M_{Z}^{2}}-\frac{A_{W W}}{M_{W}^{2}} \\
\epsilon_{2} & =F_{W W}-F_{33} \\
\epsilon_{3} & =\frac{\cos \theta}{\sin \theta} F_{30}
\end{aligned}
$$

We summarize this dependence below:

$$
\begin{aligned}
\Delta r_{W} & =-\frac{\cos ^{2} \theta}{\sin ^{2} \theta} \epsilon_{1}+\frac{\cos 2 \theta}{\sin ^{2} \theta} \epsilon_{2}+2 \epsilon_{3} \\
\Delta k & =-\frac{\cos ^{2} \theta}{\cos 2 \theta} \epsilon_{1}+\frac{1}{\cos 2 \theta} \epsilon_{3} \\
\Delta \rho & =\epsilon_{1}
\end{aligned}
$$

From the experimental values $M_{W} / M_{Z}=0.8807 \pm 0.0031, A_{F B}^{l}=0.0174 \pm 0.0030$ and $\Gamma_{l}=83.52 \pm 0.33 \mathrm{MeV}$, one finds 42]:

$$
\begin{aligned}
& \epsilon_{1}=(0.15 \pm 0.41) \cdot 10^{-2} \\
& \epsilon_{2}=(-0.71 \pm 0.83) \cdot 10^{-2} \\
& \epsilon_{3}=(-0.02 \pm 0.56) \cdot 10^{-2}
\end{aligned}
$$

As expected, the physical quantities depend on the three fundamental constants of the electroweak theory and on three additional parameters carrying, in our approximation, all the information concerning the quantum corrections 9 . In the next section we will relate these parameters to those which characterize the effective lagrangian of the electroweak interactions up to $\mathrm{O}\left(p^{4}\right)$.

\footnotetext{
${ }^{6}$ To remove the assumptions (i) and (ii) made above, one may consider the eqs. (4.38) as definitions of the parameters $\epsilon$ 's, taking advantage of the fact that the three observables involved are experimentally clean. This is the point of view advocated by the authors of ref. [40]. Then the relation between the $\epsilon$ 's and the radiative corrections will depend on the particular model examined.
} 


\section{Electroweak Interactions: Higher Orders}

In section 3, we have shown how to build an effective lagrangian for the electroweak interactions. The underlying fundamental theory might considerably differ from what presently assumed. In particular, particles much heavier than those characterizing the known low-energy spectrum might exist. In this case, none of the low-energy models described in section 3 will be able to reproduce the predictions of the theory. As we have seen in the previous section, in general, new heavy particles will affect the physical observables through their contribution to radiative corrections. The low-energy models, as specified in section 3, cannot account for these corrections and appropriate extensions of them are required.

At the same time, there is an independent motivation to enlarge the low energy models introduced up to now. In fact, the lagrangian $\mathcal{L}$ of eq. (3.33) is naturally organized in a derivative expansion, whose lowest order term is precisely given by eq. (3.33). At the next order, gauge invariant structures with up to four derivatives must be included in the effective lagrangian, and so on. As a first step, we will introduce these additional terms. To this purpose we introduce the combinations:

$$
\begin{aligned}
T & =U \tau^{3} U^{\dagger} \\
V_{\mu} & =\left(D_{\mu} U\right) U^{\dagger}
\end{aligned}
$$

They transform under $S U(2)_{L} \otimes U(1)_{Y}$ as follows:

$$
\begin{aligned}
T^{\prime} & =g_{L} T g_{L}^{\dagger} \\
V_{\mu}^{\prime} & =g_{L} V_{\mu} g_{L}^{\dagger}
\end{aligned}
$$

The covariant derivative acting on $V_{\mu}$ is given by:

$$
\mathcal{D}_{\mu} V_{\nu}=\partial_{\mu} V_{\nu}-g\left[\hat{W}_{\mu}, V_{\nu}\right]
$$

A frequently occurring identity is:

$$
\mathcal{D}_{\mu} V_{\nu}-\mathcal{D}_{\nu} V_{\mu}=-g \hat{W}_{\mu \nu}+g^{\prime} U \hat{B}_{\mu \nu} U^{\dagger}+\left[V_{\mu}, V_{\nu}\right]
$$

The algebraically independent $S U(2)_{L} \otimes U(1)_{Y}$ and CP invariants, functions of the gauge vector bosons and the Goldstone fields, containing up to four derivatives are listed below [12, 13]:

$$
\begin{aligned}
\mathcal{L}_{0} & =\frac{v^{2}}{4}\left[\operatorname{tr}\left(T V_{\mu}\right)\right]^{2} \\
\mathcal{L}_{1} & =i \frac{g g^{\prime}}{2} B_{\mu \nu} \operatorname{tr}\left(T \hat{W}^{\mu \nu}\right)
\end{aligned}
$$




$$
\begin{aligned}
\mathcal{L}_{2} & =i \frac{g^{\prime}}{2} B_{\mu \nu} \operatorname{tr}\left(T\left[V^{\mu}, V^{\nu}\right]\right) \\
\mathcal{L}_{3} & =g \operatorname{tr}\left(\hat{W}_{\mu \nu}\left[V^{\mu}, V^{\nu}\right]\right) \\
\mathcal{L}_{4} & =\left[\operatorname{tr}\left(V_{\mu} V_{\nu}\right)\right]^{2} \\
\mathcal{L}_{5} & =\left[\operatorname{tr}\left(V_{\mu} V^{\mu}\right)\right]^{2} \\
\mathcal{L}_{6} & =\operatorname{tr}\left(V_{\mu} V_{\nu}\right) \operatorname{tr}\left(T V^{\mu}\right) \operatorname{tr}\left(T V^{\nu}\right) \\
\mathcal{L}_{7} & =\operatorname{tr}\left(V_{\mu} V^{\mu}\right)\left[\operatorname{tr}\left(T V^{\nu}\right)\right]^{2} \\
\mathcal{L}_{8} & =\frac{g^{2}}{4}\left[\operatorname{tr}\left(T \hat{W}_{\mu \nu}\right)\right]^{2} \\
\mathcal{L}_{9} & =\frac{g}{2} \operatorname{tr}\left(T \hat{W}_{\mu \nu}\right) \operatorname{tr}\left(T\left[V^{\mu}, V^{\nu}\right]\right) \\
\mathcal{L}_{10} & =\left[\operatorname{tr}\left(T V_{\mu}\right) \operatorname{tr}\left(T V_{\nu}\right)\right]^{2} \\
\mathcal{L}_{11} & =\operatorname{tr}\left(\left(\mathcal{D}_{\mu} V^{\mu}\right)^{2}\right) \\
\mathcal{L}_{12} & =\operatorname{tr}\left(T \mathcal{D}_{\mu} \mathcal{D}_{\nu} V^{\nu}\right) \operatorname{tr}\left(T V^{\mu}\right) \\
\mathcal{L}_{13} & =\frac{1}{2}\left[\operatorname{tr}\left(T \mathcal{D}_{\mu} V_{\nu}\right)\right]^{2} \\
\mathcal{L}_{14} & =i g \epsilon^{\mu \nu \rho \sigma} \operatorname{tr}\left(\hat{W}_{\mu \nu} V_{\rho}\right) \operatorname{tr}\left(T V_{\sigma}\right)
\end{aligned}
$$

Before analyzing the physical meaning of the invariants $\mathcal{L}_{i}(i=0, \ldots 14)$, we will discuss the arbitrariness in the choice of a particular base of invariants. The above base can be modified either by adding total derivatives to the various terms, or by making use of the classical equations of motion [43, 33, 44].

To illustrate this last point, we consider an effective lagrangian $\mathcal{L}_{\text {eff }}$, depending on a single scalar field $\varphi$ and its derivatives, of the following form:

$$
\begin{aligned}
\mathcal{L}_{\text {eff }} & =\mathcal{L}_{c l}+\sum_{i} c_{i} \mathcal{L}_{i} \\
\mathcal{L}_{c l} & =\frac{1}{2} \partial_{\mu} \varphi \partial^{\mu} \varphi-\frac{m^{2}}{2} \varphi^{2}-V(\varphi)
\end{aligned}
$$

We assume that the coefficients $c_{i}$ are of order $h$. What we have in mind is that the part $\sum_{i} c_{i} \mathcal{L}_{i}$, together with the $\mathrm{O}(h)$ corrections from $\mathcal{L}_{c l}$, correctly reproduce the results of the underlying fundamental theory, at one-loop level.

Suppose that the term $\mathcal{L}_{j}, j$ being one particular among the $i$ indices, has the form:

$$
\mathcal{L}_{j}=\left(\partial^{2}+m^{2}\right) \varphi \cdot F(\varphi)
$$

where $F(\varphi)$ is at least quadratic in $\varphi$ and/or its derivatives. We perform the following local transformation on the field $\varphi$ :

$$
\delta \varphi=c_{j} F(\varphi)
$$


Notice that this is an allowed transformation, so that the S-matrix elements do not change. One obtains:

$$
\begin{aligned}
\delta S_{c l} & =\int d x \frac{\delta \mathcal{L}_{c l}}{\delta \varphi} \delta \varphi \\
& =-\int d x\left[\left(\partial^{2}+m^{2}\right) \varphi+\frac{\delta V}{\delta \varphi}\right] c_{j} F(\varphi) \\
& =-\int d x c_{j} \mathcal{L}_{j}-\int d x c_{j} \frac{\delta V}{\delta \varphi} F(\varphi)
\end{aligned}
$$

On the other hand:

$$
\delta\left(\int d x \sum_{i} c_{i} \mathcal{L}_{i}\right)=O\left(h^{2}\right)
$$

In conclusion the transformed lagrangian is given by:

$$
\mathcal{L}_{\text {eff }}^{\prime}=\mathcal{L}_{c l}+\sum_{i \neq j} c_{i} \mathcal{L}_{i}-c_{j} \frac{\delta V}{\delta \varphi} F(\varphi)+O\left(h^{2}\right)
$$

The net effect of the transformation given in eq. (5.9) is identical to that obtained by using the classical equations of motion. Up to higher order corrections, $\mathcal{L}_{\text {eff }}$ and $\mathcal{L}_{\text {eff }}^{\prime}$ will give rise to the same on-shell amplitudes ๆ. In the following we will make use of this freedom in order to isolate the physically independent effects related to the invariant structures listed in eq. (5.5).

Coming back to the lagrangian $\mathcal{L}$ of eq. (3.33), the equations of motion for the gauge fields are given by:

$$
\begin{gathered}
\partial^{\mu} B_{\mu \nu}+i g^{\prime} \frac{v^{2}}{4} \operatorname{tr}\left(T V_{\nu}\right)-g^{\prime} J_{\nu}^{B}=0 \\
\mathcal{D}^{\mu} \hat{W}_{\mu \nu}-g \frac{v^{2}}{4} V_{\nu}-\frac{g}{2 i} \hat{J}_{\nu}^{W}=0
\end{gathered}
$$

where:

$$
\begin{aligned}
J_{\mu}^{B} & =J_{\mu}^{(B-L)}+J_{\mu}^{3 R} \\
& =\sum_{\psi} \bar{\psi}_{L} \gamma^{\mu} \frac{(B-L)}{2} \psi_{L}+\sum_{\psi} \bar{\psi}_{R} \gamma^{\mu} \frac{\tau^{3}+(B-L)}{2} \psi_{R} \\
\hat{J}_{\mu}^{W} & =\sum_{\psi}\left(\bar{\psi}_{L} \gamma^{\mu} \frac{\tau^{a}}{2} \psi_{L}\right) \tau^{a}
\end{aligned}
$$

\footnotetext{
${ }^{7}$ The result generalizes to higher orders 43 . If the effective theory contains terms up to the order $O\left(h^{n}\right)$, and if the kinetic operator $\left(\partial^{2}+m^{2}\right)$ has already been eliminated from all the terms of order $O\left(h^{m}\right) \quad(m<n)$, then the use of the classical equation of motion gives rise to an equivalent effective action.
} 
- Exercise: derive eqs. (5.13-5.16).

Since $\partial_{\mu} \partial_{\nu} B^{\mu \nu}=0$ and $\mathcal{D}_{\mu} \mathcal{D}_{\nu} \hat{W}^{\mu \nu}=0$, from eqs. (5.13-5.14), one obtains:

$$
\begin{aligned}
\partial_{\nu} \operatorname{tr}\left(T V^{\nu}\right) & =-i \frac{4}{v^{2}} \partial^{\nu} J_{\nu}^{B} \\
\mathcal{D}^{\nu} V_{\nu} & =i \frac{2}{v^{2}} \mathcal{D}^{\nu} \hat{J}_{\nu}^{W}
\end{aligned}
$$

Both right-hand sides of eqs. (5.17) and (5.18) are classically given by expressions of the kind:

$$
\sum_{\psi} \frac{m_{\psi}}{v^{2}}(\bar{\psi} \psi)
$$

Therefore, as long as one considers light fermions, $m_{\psi} \ll v$, they are practically negligible and they will be discarded from now on.

- Exercise: by using the equations of motion for the gauge fields $W$ and $B$, given in eqs. (5.13-5.18), show that:

$$
\begin{aligned}
& \mathcal{L}_{11}=0 \\
& \mathcal{L}_{12}=0 \\
& \mathcal{L}_{13}=-\frac{g^{\prime 2}}{4} B_{\mu \nu} B^{\mu \nu}+\mathcal{L}_{1}+\mathcal{L}_{8}-\mathcal{L}_{4}+\mathcal{L}_{5}-\mathcal{L}_{6}+\mathcal{L}_{7}
\end{aligned}
$$

As a consequence of the relations (5.20-5.22), one has the equivalence among the effective lagrangians:

$$
\mathcal{L}_{\text {eff }}=\mathcal{L}+\sum_{i=0}^{14} a_{i} \mathcal{L}_{i}
$$

and

$$
\mathcal{L}_{\text {eff }}^{\prime}=\hat{\mathcal{L}}+\sum_{i=0}^{14} \hat{a}_{i} \mathcal{L}_{i}
$$

with:

$$
\begin{aligned}
& \hat{a}_{1}=a_{1}+a_{13} \\
& \hat{a}_{4}=a_{4}-a_{13} \\
& \hat{a}_{5}=a_{5}+a_{13} \\
& \hat{a}_{6}=a_{6}-a_{13} \\
& \hat{a}_{7}=a_{7}+a_{13} \\
& \hat{a}_{8}=a_{8}+a_{13} \\
& \hat{a}_{11}=0 \\
& \hat{a}_{12}=0 \\
& \hat{a}_{13}=0
\end{aligned}
$$


For the coefficients not listed above one has $\hat{a}_{i}=a_{i}$ and the lagrangian $\hat{\mathcal{L}}$ differs from $\mathcal{L}$ by a wave function renormalization for the vector boson $B$. 


\section{$6 \quad$ Physical Meaning of $\mathcal{L}_{\text {eff }}$}

We are now ready to discuss the meaning of the base $\left\{\mathcal{L}_{i}\right\}$ given in eq (5.5). The physical content of the base is more transparent in the unitary gauge, $U=\mathbf{1}$, where all the invariants $\mathcal{L}_{i}$ collapse into polynomials of the gauge fields. All the invariants contain at most quartic terms in the gauge fields, but they can be grouped appropriately, depending on their expansion which can start from two, three or four gauge vector bosons. The structures containing bilinear terms in the gauge fields are just six:

$$
\begin{aligned}
a_{0} \mathcal{L}_{0} & =-\frac{1}{4} a_{0} v^{2}\left(g W_{\mu}^{3}-g^{\prime} B_{\mu}\right)^{2}+\ldots \\
a_{1} \mathcal{L}_{1} & =\frac{1}{2} a_{1} g g^{\prime} B_{\mu \nu}\left(\partial^{\mu} W^{3^{\nu}}-\partial^{\nu} W^{3^{\mu}}\right)+\ldots \\
a_{8} \mathcal{L}_{8} & =-\frac{1}{4} a_{8} g^{2}\left(\partial_{\mu} W^{3}{ }_{\nu}-\partial_{\nu} W^{3}{ }_{\mu}\right)^{2}+\ldots \\
a_{11} \mathcal{L}_{11} & =-\frac{1}{2} a_{11}\left[g^{2}\left(\partial_{\mu} W^{1^{\mu}}\right)^{2}+g^{2}\left(\partial_{\mu} W^{2^{\mu}}\right)^{2}+\left(\partial_{\mu}\left(g W^{3^{\mu}}-g^{\prime} B^{\mu}\right)\right)^{2}\right]+\ldots \\
a_{12} \mathcal{L}_{12} & =-a_{12} \partial_{\mu} \partial_{\nu}\left(g W^{3^{\nu}}-g^{\prime} B^{\nu}\right) \cdot\left(g W^{3^{\mu}}-g^{\prime} B^{\mu}\right)+\ldots \\
a_{13} \mathcal{L}_{13} & =-\frac{1}{2} a_{13} \partial_{\mu}\left(g W^{3}{ }_{\nu}-g^{\prime} B_{\nu}\right) \cdot \partial^{\mu}\left(g W^{3^{\nu}}-g^{\prime} B^{\nu}\right)+\ldots
\end{aligned}
$$

The dots stand for trilinear and quadrilinear terms in the gauge vector bosons. They are there, together with the terms containing the would-be Goldstone bosons to ensure the gauge invariance of each structure. It is straightforward to compute the contributions of the above terms to the various two-point functions. We obtain:

$$
\begin{aligned}
-i \Pi_{\mu \nu}^{11}= & -i a_{11} g^{2} p_{\mu} p_{\nu} \\
-i \Pi_{\mu \nu}^{33}= & -\frac{i}{2} a_{0} v^{2} g^{2} g_{\mu \nu} \\
& -i\left(a_{8}+a_{13}\right) g^{2}\left(p^{2} g_{\mu \nu}-p_{\mu} p_{\nu}\right) \\
& -i\left(a_{11}-2 a_{12}+a_{13}\right) g^{2} p_{\mu} p_{\nu} \\
-i \Pi_{\mu \nu}^{30}= & \frac{i}{2} a_{0} v^{2} g g^{\prime} g_{\mu \nu} \\
& +i\left(a_{1}+a_{13}\right) g g^{\prime}\left(p^{2} g_{\mu \nu}-p_{\mu} p_{\nu}\right) \\
& +i\left(a_{11}-2 a_{12}+a_{13}\right) g g^{\prime} p_{\mu} p_{\nu} \\
-i \Pi_{\mu \nu}^{00}= & -\frac{i}{2} a_{0} v^{2} g^{\prime 2} g_{\mu \nu} \\
& -i a_{13} g^{\prime 2}\left(p^{2} g_{\mu \nu}-p_{\mu} p_{\nu}\right) \\
& -i\left(a_{11}-2 a_{12}+a_{13}\right) g^{\prime 2} p_{\mu} p_{\nu}
\end{aligned}
$$


One may be surprised by the fact that, apparently, beyond the lowest order represented by the lagrangian $\mathcal{L}$, the gauge-invariant independent terms affecting the two-point functions of the theory are six and not three as expected on the basis of our discussion about the radiative corrections. Notice that, as already observed, the present discussion is also based on an expansion in powers of momenta where we are keeping exactly the same order as in eq. (4.3). The apparent paradox is solved by recalling that, with the use of the equations of motion, $\mathcal{L}_{11}, \mathcal{L}_{12}$ and $\mathcal{L}_{13}$ can be eliminated by suitably redefining the parameters of the effective lagrangian, that is by using the effective theory $\mathcal{L}_{\text {eff }}^{\prime}$ defined in eqs. (5.24-5.25). If we insist in using the complete base, with non-vanishing $a_{11}, a_{12}$ and $a_{13}$, things will arrange in such a way that the physical quantities will depend only on three combinations among the six parameters $a_{0}, a_{1}, a_{8}, a_{11}, a_{12}$ and $a_{13}$. This is already evident from eqs. (6.2): the parameters $a_{11}$ and $a_{12}$ always multiply the harmless terms proportional to $p_{\mu} p_{\nu}$ and the parameter $a_{13}$ can be absorbed in a redefinition of $\alpha$ and $M_{Z}$.

- Exercise: compute the contribution of $\mathcal{L}_{\text {eff }}=\mathcal{L}+\sum_{i=0}^{14} a_{i} \mathcal{L}_{i}$ to the parameters $\epsilon_{1}, \epsilon_{2}$ and $\epsilon_{3}$. One finds:

$$
\begin{aligned}
\epsilon_{1} & =2 a_{0} \\
\epsilon_{2} & =-g^{2}\left(a_{8}+a_{13}\right) \\
\epsilon_{3} & =-g^{2}\left(a_{1}+a_{13}\right)
\end{aligned}
$$

The above relations show that the coefficients $a_{0},\left(a_{8}+a_{13}\right)$ and $\left(a_{1}+a_{13}\right)$ are directly related to the observables discussed in section 4 , and are therefore appropriate in parametrizing precision measurements performed at LEP I.

The other terms of the base $\mathcal{L}_{i}(i=0,14)$ can be discussed along similar lines. The invariants whose expansion starts with three gauge fields are $\mathcal{L}_{2}, \mathcal{L}_{3}, \mathcal{L}_{9}$ and $\mathcal{L}_{14}$. Again, the use of the equations of motion can help in analyzing the physical effects. It turns out that [10]:

$$
\begin{aligned}
& \mathcal{L}_{2}=i g^{\prime 2} J_{\mu}^{B} \operatorname{tr}\left(T V^{\mu}\right)+\left(\text { redefinitions of } \delta Z_{1}, a_{0}, a_{1}\right) \\
& \mathcal{L}_{3}=i g^{2} \operatorname{tr}\left(\hat{J}_{\mu}^{W} V^{\mu}\right)+\left(\text { redefinitions of } \delta v^{2}, \delta Z_{2}, a_{1}\right)
\end{aligned}
$$

The parameters $\delta Z_{1}, \delta Z_{2}$ and $\delta v^{2}$ correspond to wave function renormalizations for the fields $B_{\mu}, W_{\mu}$ and $\xi$, respectively. The equations (6.4) bring in new invariant structures, not considered up to now, containing fermionic vertices. In this new base one would have universal corrections to the fermionic vertices, leading however to the same physical predictions obtained in the framework of the original, purely bosonic base. 
The parameters $a_{2}, a_{3}, a_{9}$ and $a_{14}$ might be useful in discussing the LEP II physics. Indeed, one can parametrize the most general couplings of two charged vector bosons with a neutral vector boson according to the effective lagrangian [45]:

$$
\begin{aligned}
\frac{\mathcal{L}_{W W N}}{g_{W W N}}= & i g_{1}{ }^{N}\left(W^{\dagger}{ }_{\mu \nu} W^{\mu} N^{\nu}-W_{\mu \nu} W^{\dagger^{\mu}} N^{\nu}\right)+i k_{N} W^{\dagger}{ }_{\mu} W_{\nu} N^{\mu \nu} \\
& +i \frac{\lambda_{N}}{M_{W}^{2}} W^{\dagger}{ }_{\lambda \mu} W^{\mu}{ }_{\nu} N^{\nu \lambda}+g_{5}^{N} \epsilon^{\mu \nu \rho \sigma}\left(W^{\dagger}{ }_{\mu} \partial_{\rho} W_{\nu}-\partial_{\rho} W^{\dagger}{ }_{\mu} W_{\nu}\right) N_{\sigma}
\end{aligned}
$$

Here $N_{\mu}$ stands for the photon $A$ or the $Z$ field, $W_{\mu}$ is the $W^{-}$field, $W_{\mu \nu}=$ $\partial_{\mu} W_{\nu}-\partial_{\nu} W_{\mu}$ and similarly for $N_{\mu \nu}$. The four terms in the previous equation are the most general $C P$ invariant terms one can build out of vector fields with vanishing divergence. The first three couplings are separately invariant under $P$ and $C$ transformations, whereas the last one violates both $P$ and $C$. Additional terms can be added if $C P$ violation is allowed [45]. The conventional choice for the overall normalization constants $g_{W W N}(N=\gamma, Z)$ is:

$$
\begin{aligned}
g_{W W \gamma} & =-e \\
g_{W W Z} & =-g \cos \theta
\end{aligned}
$$

The first term in eq. (6.5) has the form of a minimal coupling of the charged $W$ current to the photon or the $Z$ field. The parameters $g_{1}^{\gamma}$ and $g_{1}^{Z}$ represent the electromagnetic and " $Z$ " charges of the $W$, in units of $g_{W W \gamma}$ and $g_{W W Z}$, respectively. The coefficients $k_{N}$ and $\lambda_{N}$ are related to the "magnetic" moments of the $W$, an anomalous "magnetic" moment occurring if $k_{N} \neq 1$ or $\lambda_{N} \neq 0$.

A direct access to the above parameters will be provided by $W$ pair production in $e^{+} e^{-}$collisions at the future LEP II facility. The differential angular distribution of the produced $W$ 's turns out to be particularly sensitive to the chosen set of $C P$ conserving couplings [45].

By identifying the trilinear gauge boson interaction terms in the effective lagrangian $\mathcal{L}_{\text {eff }}$, eq. (5.23), we can readily express the coefficients of $\mathcal{L}_{W W N}$ in terms of the parameters $a_{i}$. One obtains:

$$
\begin{aligned}
g_{1}^{\gamma}-1 & =0 \\
g_{1}^{Z}-1 & =-\frac{g^{2}}{\cos ^{2} \theta} a_{3} \\
k_{\gamma}-1 & =g^{2}\left(a_{2}-a_{3}-a_{9}\right)-\epsilon_{2}+\epsilon_{3} \\
k_{Z}-1 & =\frac{g^{2}}{\cos ^{2} \theta}\left[\cos ^{2} \theta\left(-a_{3}-a_{9}\right)-\sin ^{2} \theta a_{2}\right]-\epsilon_{2}-\tan ^{2} \theta \epsilon_{3} \\
\lambda_{\gamma} & =0 \\
\lambda_{Z} & =0
\end{aligned}
$$




$$
\begin{aligned}
g_{5}^{\gamma} & =0 \\
g_{5}^{Z} & =-\frac{g^{2}}{\cos ^{2} \theta} a_{14}
\end{aligned}
$$

The relation $g_{1}^{\gamma}=1$ expresses the exact conservation of the electromagnetic charge. The coefficients $\lambda_{N}$ are vanishing since $\mathcal{L}_{\text {eff }}$ contains terms up to the fourth order in momenta or gauge fields.

The parameters $a_{2}, a_{3}$ and $a_{9}$ or any set of independent combinations, together with the coefficient $a_{14}$, appear to play in LEP II physics the same role covered by the $\epsilon$ 's parameters in LEP I measurements. Notice that invariance under isospin would require $a_{9}=a_{14}=0$.

It may be observed that the present bounds on the $\epsilon$ 's parameters, summarized in eq. (4.39), translate into a bound of few percents on $a_{0},\left(a_{8}+a_{13}\right)$ and $\left(a_{1}+a_{13}\right)$. On the other hand, it seems reasonable to assume that, in any sensible theory, the parameters $a_{i}$ are all of the same order of magnitude. It would be a rather surprising result to find that, for instance, $a_{2}$ and $a_{3}$ are larger than $a_{0},\left(a_{8}+a_{13}\right)$ and $\left(a_{1}+a_{13}\right)$ by a factor 10 or more. On this basis, one would expect that the deviations listed in eq. (6.7) might be at most of order $10^{-2}-10^{-1}$, and thus probably too small to be appreciated at LEP II [10]. Indeed, if we were to use "naive dimensional analysis" 25] to estimate the size of the effects at LEP II, by assuming a range of validity for $\mathcal{L}_{\text {eff }}$ extending up to energies close to $4 \pi v$, we would guess for the dimensionless coefficients $a_{i}$ values of order one in units of $\left(1 / 16 \pi^{2}\right)$, even smaller than those indicated by the LEP I data.

It is maybe useful to recall that this kind of considerations requires that the scale of new physics is considerably higher than $2 M_{W}$ and, in any event, it is certainly not a substitute for the experimental tests [46].

Finally, $\mathcal{L}_{4}, \mathcal{L}_{5}, \mathcal{L}_{6}, \mathcal{L}_{7}$ and $\mathcal{L}_{10}$ contain only quadrilinear terms in the gauge boson fields. We could think to fix them, at least in principle, by means of scattering experiments among gauge vector bosons, to occur at the future LHC/SSC facilities [47]. Notice that all the invariants but $\mathcal{L}_{14}$ are invariant under parity. 


\section{The Matching Conditions}

The effective lagrangian $\mathcal{L}_{\text {eff }}$ defined in the previous section is able to reproduce the quantum effects originating from the heavy sector of an underlying fundamental theory of the electroweak interactions. Such a piece of information is contained in the coefficients $a_{i}$ of the invariants $\mathcal{L}_{i}$. In particular, $a_{0}, a_{1}, a_{8}, a_{11}, a_{12}$ and $a_{13}$ are related, as we have seen, to the self-energy corrections. Corrections to the three and four point functions may be discussed as well.

Before concluding these lectures, we will show, in an example, how to deduce, in practice, the coefficients $a_{i}$ from the knowledge of the fundamental theory. This is done by means of the so called matching procedure [24]. It consists in equating amplitudes evaluated in the fundamental theory and in the effective one. At the end, the parameters of the effective theory will be run from the scale where the matching has taken place, down to the energy where one will use the effective lagrangian.

As a simple example, we consider a fundamental theory consisting just of the standard model with a fourth generation of light leptons $\left(M_{l} \leq M_{Z}\right)$ and heavy quarks $\left(M \gg M_{Z}\right)$. We denote by $M$ the common mass of the quarks. We will restrict the discussion to the two point functions in the gauge boson sector evaluated at one-loop order, the extension to the other Green functions being straightforward p.

The matching condition is given by:

$$
\Pi_{C \mu \nu}^{i j}+\Pi_{L \mu \nu}^{i j}=\Pi_{\hat{C}}^{i j}+\Pi_{a \mu \nu}^{i j}
$$

Here the left-hand side refers to the fundamental theory and the right-hand side refers to the effective one. $\Pi_{C}$ is the contribution of the counterterms of the fundamental theory, derived from:

$$
\begin{gathered}
-\frac{1}{4}\left(1+\delta Z_{1}\right) B_{\mu \nu} B^{\mu \nu}-\frac{1}{4}\left(1+\delta Z_{2}\right) W_{\mu \nu}^{i} W^{i \mu \nu}+ \\
+\frac{1}{8}\left(v^{2}+\delta v^{2}\right) g^{2}\left(W_{\mu}^{1} W^{1 \mu}+W_{\mu}^{2} W^{2 \mu}\right)+\frac{1}{8}\left(v^{2}+\delta v^{2}\right)\left(g W_{\mu}^{3}-g^{\prime} B_{\mu}\right)^{2}
\end{gathered}
$$

$\Pi_{L}$ is the loop contribution from the heavy quark doublet. $\Pi_{a}$ is the contribution from the invariants $\mathcal{L}_{i}$, already evaluated in eq. (6.2). Finally, $\Pi_{\hat{C}}$ is the contribution from possible finite counterterms in the effective theory, of exactly the same form as those given in eq. (7.2), with renormalization constants $\delta \hat{Z}_{1}, \delta \hat{Z}_{2}, \delta \hat{v}^{2}$. Such terms

\footnotetext{
${ }^{8}$ The effective theory will also contain a Wess-Zumino term [21] whose gauge variation cancels the anomaly produced by the light leptons [16].
} 
may be present for a particular choice of renormalization conditions. Two comments are in order.

We have not included the loop contributions from the light sector. These are exactly the same for the fundamental and the effective theories, and can be simply dropped out from both sides of eq. (7.1). Actually, this is the reason why at low energy we can define an effective lagrangian. The amplitude computed in the fundamental theory in the large mass limit and the amplitude evaluated with the lagrangian obtained by simply suppressing the heavy fields, have equal absorptive parts in the various channels. Their difference is an analytic function, which, for momenta much lower than the scale $M$, can be approximated with a polynomial.

In principle, in a generic renormalization scheme, additional finite counterterms have to be added to the loop contribution from the fundamental theory, to properly ensure the validity of the Ward identities. If the regularization procedure does not respect the gauge symmetry, the Ward identities will be apparently broken by a loop computation and they will have to be repaired by properly adding finite terms. In the following, we will use dimensional regularization with $\left\{\gamma_{\mu}, \gamma_{5}\right\}=0$ in all dimensions, which automatically accounts for the non-anomalous Ward identities and which, in the example given, can be safely applied.

The contributions from the counterterms of eq. (7.2) are given by:

$$
\begin{aligned}
-i \Pi_{C \mu \nu}^{11}=-i \Pi_{C \mu \nu}^{22}= & -i \Pi_{C \mu \nu}^{33}=i \frac{\delta v^{2}}{4} g^{2} g_{\mu \nu}-i \delta Z_{2}\left(p^{2} g_{\mu \nu}-p_{\mu} p_{\nu}\right) \\
-i \Pi_{C}^{30} & =-i \frac{\delta v^{2}}{4} g g^{\prime} g_{\mu \nu} \\
& -i \Pi_{C \mu \nu}^{00}=i \frac{\delta v^{2}}{4} g^{\prime 2} g_{\mu \nu}-i \delta Z_{1}\left(p^{2} g_{\mu \nu}-p_{\mu} p_{\nu}\right)
\end{aligned}
$$

Those from the counterterms of the effective theory are immediately derived from the above equations. Finally, the one-loop contribution from the heavy quark doublet is given by:

$$
\begin{aligned}
-i \Pi_{L \mu \nu}^{11}=-i \Pi_{L \mu \nu}^{22}=-i \Pi_{L \mu \nu}^{33}= & \frac{-i}{16 \pi^{2}} g^{2}\left\{3 M^{2}\left(A+\ln \frac{M^{2}}{\mu^{2}}\right) g_{\mu \nu}\right. \\
& +\left[-\left(A+\ln \frac{M^{2}}{\mu^{2}}\right)-\frac{1}{2}\right]\left(p^{2} g_{\mu \nu}-p_{\mu} p_{\nu}\right) \\
& \left.-\frac{1}{2} p_{\mu} p_{\nu}\right\} \\
-i \Pi_{L \mu \nu}^{30}= & \frac{-i}{16 \pi^{2}} g g^{\prime}\left\{-3 M^{2}\left(A+\ln \frac{M^{2}}{\mu^{2}}\right) g_{\mu \nu}\right.
\end{aligned}
$$




$$
\begin{aligned}
& +\frac{1}{2}\left(p^{2} g_{\mu \nu}-p_{\mu} p_{\nu}\right) \\
& \left.+\frac{1}{2} p_{\mu} p_{\nu}\right\} \\
-i \Pi_{L_{\mu \nu}^{00}}^{00}= & \frac{-i}{16 \pi^{2}} g^{2}\left\{3 M^{2}\left(A+\ln \frac{M^{2}}{\mu^{2}}\right) g_{\mu \nu}\right. \\
& +\left[-\frac{11}{9}\left(A+\ln \frac{M^{2}}{\mu^{2}}\right)-\frac{1}{2}\right]\left(p^{2} g_{\mu \nu}-p_{\mu} p_{\nu}\right) \\
& \left.-\frac{1}{2} p_{\mu} p_{\nu}\right\}
\end{aligned}
$$

where

$$
\begin{aligned}
A & =-\frac{2}{4-d}+\gamma_{E}-\ln 4 \pi \\
\gamma_{E} & \simeq 0.577
\end{aligned}
$$

and $\mu$ denotes the scale parameter of dimensional regularization. The expressions given above have been obtained in the limit $p^{2} \ll M^{2}$, neglecting positive powers of $p^{2} / M^{2}$. We are now ready to solve the matching conditions. From eq. (7.1), by using eqs. (7.3-7.6) and (6.2), one obtains:

$$
\begin{aligned}
\delta v^{2}-\delta \hat{v}^{2} & =\frac{1}{16 \pi^{2}} 12 M^{2}\left(A+\ln \frac{M^{2}}{\mu^{2}}\right) \\
\delta Z_{2}-\delta \hat{Z}_{2} & =\frac{g^{2}}{16 \pi^{2}}\left(A+\ln \frac{M^{2}}{\mu^{2}}+\frac{1}{2}\right) \\
\delta Z_{1}-\delta \hat{Z}_{1} & =g^{\prime 2} a_{13}+\frac{g^{\prime 2}}{16 \pi^{2}}\left[\frac{11}{9}\left(A+\ln \frac{M^{2}}{\mu^{2}}\right)+\frac{1}{2}\right]
\end{aligned}
$$

and

$$
\begin{aligned}
a_{0} & =0 \\
a_{1}+a_{13} & =\frac{1}{16 \pi^{2}}\left(-\frac{1}{2}\right) \\
a_{8}+a_{13} & =0 \\
a_{11} & =\frac{1}{16 \pi^{2}}\left(-\frac{1}{2}\right) \\
a_{13} & =2 a_{12}
\end{aligned}
$$

As expected, these relations are already sufficient to determine the contribution of the heavy quark doublet to the parameters $\epsilon$ 's. One finds [48]:

$$
\begin{aligned}
\delta \epsilon_{1} & =0 \\
\delta \epsilon_{2} & =0 \\
\delta \epsilon_{3} & =+\frac{g^{2}}{32 \pi^{2}}
\end{aligned}
$$


Notice that, since the parameters $\epsilon_{1}$ and $\epsilon_{2}$ are associated to isospin breaking effects (see eqs. (4.37)), they receive a vanishing contribution from a degenerate quark doublet.

- Exercise: compute the contribution to the $\epsilon$ 's parameters from a degenerate doublet of heavy leptons. Do the overall contribution to $\epsilon_{3}$ due to a whole fourth generation of heavy degenerate fermions vanish?

The case analyzed above provides an example of non-decoupling. If decoupling applies, the effects of a large mass limit are just the appearance of higher dimensional operators, with coefficients suppressed by inverse powers of the large mass, and a renormalization of the parameters [15]. In this case the physics associated with the heavy scale decouples from the low energy theory. Instead, the effects described in eqs. (7.9)-(7.10) are neither suppressed nor absorbable in a redefinition of the fundamental constants. The point is that, in order to have decoupling, one is not allowed to let a dimensionless coupling grow with the heavy mass. On the other hand, this is just our case, since, in order to preserve the gauge invariance, the large mass limit for the quark doublet has been implicitly achieved by increasing the corresponding Yukawa coupling. P

By imposing the matching conditions on a sufficient number of gauge bosons Green functions, one can determine the whole set of parameters $a_{i}(i=0, \ldots 14)$ and we have collected the results in table III.

\footnotetext{
9 The effective lagrangian we have dealt with so far has been constructed by applying the formalism of non-linear realizations sketched in section 2. According to a widely accepted point of view, going beyond the SM, one should use non-linear realizations whenever the decoupling theorem does not apply, the opposite case requiring the use of linear realizations. Although this choice may be in practice convenient, it is not so compelling. In fact, the use of non-linear realizations is requested when the degrees of freedom of the low energy effective theory cannot be assembled into linear multiplets of the symmetry group $G$. When this happens the low energy theory is non-renormalizable, which is indeed the case if the decoupling theorem does not apply. On the other hand, when the light degrees of freedom can be arranged into linear multiplets of $G$, which may happen whether or not the decoupling theorem applies, one can choose to work with linear realizations or non-linear ones, the two being related by an allowed field transformation. For instance, the case of a vector-like doublet of heavy quarks can still be discussed in the framework of the effective lagrangian of eq. (5.6). In this case, the effects of the doublet decouple and the coefficients $a_{i}$ will contain inverse power of the heavy scale [49].
} 


\begin{tabular}{|c|c|c|c|}
\hline & $\begin{array}{l}\text { LARGE } m_{\sigma} \llbracket 13 \\
L=\ln \left(m_{\sigma} / \mu\right)\end{array}$ & LARGE $m_{t^{\prime}}=m_{b^{\prime}}=M$ 16 & $\begin{array}{c}\text { LARGE } m_{t} \gg m_{b} \sqrt{18} \\
L=\ln \left(m_{t} / \mu\right)\end{array}$ \\
\hline$a_{0}$ & $-\frac{3}{4} g^{\prime 2} L$ & 0 & $\frac{3}{2} \frac{m_{t}^{2}}{v^{2}}$ \\
\hline$a_{1}$ & $-\frac{1}{6} L$ & $-\frac{1}{2}$ & $+\frac{1}{3} L-\frac{1}{4}$ \\
\hline$a_{2}$ & $-\frac{1}{12} L$ & $-\frac{1}{2}$ & $+\frac{1}{3} L-\frac{3}{4}$ \\
\hline$a_{3}$ & $+\frac{1}{12} L$ & $+\frac{1}{2}$ & $+\frac{3}{8}$ \\
\hline$a_{4}$ & $+\frac{1}{6} L$ & $+\frac{1}{4}$ & $+L-\frac{5}{6}$ \\
\hline$a_{5}$ & $+\frac{1}{12} L$ & $-\frac{1}{8}$ & $-L+\frac{23}{24}$ \\
\hline$a_{6}$ & 0 & 0 & $-L+\frac{23}{24}$ \\
\hline$a_{7}$ & 0 & 0 & $+L-\frac{23}{24}$ \\
\hline$a_{8}$ & 0 & 0 & $+L-\frac{7}{12}$ \\
\hline$a_{9}$ & 0 & 0 & $+L-\frac{23}{24}$ \\
\hline$a_{10}$ & 0 & 0 & $-\frac{1}{64}$ \\
\hline$a_{11}$ & 0 & $-\frac{1}{2}$ & $-\frac{1}{2}$ \\
\hline$a_{12}$ & 0 & 0 & $-\frac{1}{8}$ \\
\hline$a_{13}$ & 0 & 0 & $-\frac{1}{4}$ \\
\hline$a_{14}$ & 0 & 0 & $+\frac{3}{8}$ \\
\hline
\end{tabular}

Table III: $a_{i}$ coefficients - in units of $\left(1 / 16 \pi^{2}\right)$ - for three particular limits of the SM. The scale $\mu$ is some intermediate scale between the low external momenta and the large mass.

As a last step, to fully define the low energy effective lagrangian, one has to specify the renormalization group equations (RGE) according to which the various parameters run from energy scales close to $M$ down to lower energies. By working in the effective theory and by choosing vanishing renormalization constants $\delta \hat{Z}_{1}$, $\delta \hat{Z}_{2}$ and $\delta \hat{v}^{2}$, one can readily conclude that no contribution from the heavy quark doublet survives in the RGE's, at one-loop order. The one-loop RGE's are then determined by the contribution of the light sector. We find instructive to recover 
this conclusion by working at the level of the fundamental theory, focusing just on the possible contribution from the heavy particles. As an example, we will compute such a contribution for the $g$ gauge coupling constant, for the previously analyzed case of a new doublet of heavy quarks. As far as the fermionic contribution is concerned, the relation between the unrenormalized coupling $g_{0}$ and the renormalized one $g$ is given by:

$$
g_{0}=\mu^{\frac{4-d}{2}}\left(\frac{g}{\sqrt{Z_{2}}}\right)
$$

where $Z_{2}=1+\delta Z_{2}$ and, since we are interested in the running of $g$ due to the heavy quark doublet, $\delta Z_{2}$ is the renormalization constant given in eq. (7.8). Actually, eq. (7.8) gives the combination $\delta Z_{2}-\delta \hat{Z}_{2}$ and, to proceed, we have to specify the finite counterterm $\delta \hat{Z}_{2}$, that is the renormalization scheme we are going to adopt. For instance, in the $\overline{\mathrm{MS}}$ scheme, we would choose $\delta \hat{Z}_{2}$ in such a way to obtain $\delta Z_{2}=\left(g^{2} / 16 \pi^{2}\right) A$, a mass independent renormalization constant. Here we prefer to make the unusual but simpler choice $\delta \hat{Z}_{2}=0$, to obtain:

$$
\delta Z_{2}=\frac{g^{2}}{16 \pi^{2}}\left(A+\ln \frac{M^{2}}{\mu^{2}}+\frac{1}{2}\right)
$$

To compute the contribution of the heavy quark doublet to the $\beta$ function of $g$, we act on both sides of eq. (7.11) with $\mu d / d \mu$. We find:

$$
0=\frac{4-d}{2}\left(\frac{g}{\sqrt{Z_{2}}}\right)+\frac{\partial}{\partial g}\left(\frac{g}{\sqrt{Z_{2}}}\right) \mu \frac{d}{d \mu} g+\mu \frac{\partial}{\partial \mu}\left(\frac{g}{\sqrt{Z_{2}}}\right)+\frac{\partial}{\partial M}\left(\frac{g}{\sqrt{Z_{2}}}\right) \mu \frac{d}{d \mu} M
$$

By explicitly evaluating the right-hand side of the previous equation and by taking the limit $d \rightarrow 4$, we obtain:

$$
\begin{aligned}
\beta(g) & =\mu \frac{d}{d \mu} g \\
& =\frac{g^{3}}{16 \pi^{2}} \frac{1}{M}\left(\mu \frac{d}{d \mu} M\right)+O\left(g^{5}\right)
\end{aligned}
$$

Eq. (7.14) shows that the usual contribution from the quark doublet, $g^{3} / 16 \pi^{2}$, drops from the final result, which is of higher order in $g$. More precisely, this cancellation is due to the term proportional to $\mu \frac{\partial}{\partial \mu}\left(\frac{g}{\sqrt{Z_{2}}}\right)$ in eq. (7.13). Usually, in a mass independent renormalization scheme, this term and the last one of eq. (7.13), are absent. On the other hand, the renormalization scheme chosen here is particularly suitable to discuss the effect of the heavy doublet, since in this case the effect simply disappears (at the order $g^{3}$ ), as expected. 
Indeed, nothing prevents the use of a mass independent scheme. For instance, in the $\overline{\mathrm{MS}}$ scheme one has $\delta Z_{2}=\left(g^{2} / 16 \pi^{2}\right) A$ and we would have obtain:

$$
\beta(g)=\frac{g^{3}}{16 \pi^{2}},
$$

the usual result for a quark doublet. However, for the matching conditions to hold, this would have required the presence of the finite counterterm:

$$
\delta \hat{Z}_{2}=-\frac{g^{2}}{16 \pi^{2}}\left(\ln \frac{M^{2}}{\mu^{2}}+\frac{1}{2}\right)
$$

In turn, this finite counterterm could have been absorbed in the effective coupling:

$$
g^{*}=g\left(1+\frac{g^{2}}{32 \pi^{2}}\left(\ln \frac{M^{2}}{\mu^{2}}+\frac{1}{2}\right)\right)
$$

As one may easily verify, $g^{*}$ runs according to the $\beta$ function defined in eq. (7.14), not containing any $g^{3}$ term.

Other cases can be studied along similar lines. The invariant structures $\mathcal{L}_{i}$ also occur in the low energy effective lagrangian derived from the standard electroweak theory in the limit of an heavy Higgs [12, 13] and in the limit of an heavy top quark [18] and we have listed the corresponding results in table III. In principle, any extension of the standard model, characterized by heavy particles, will leave its peculiar mark at low energies through a specific contribution to the parameters $a_{i}$.

ACKNOWLEDGMENT: I would like to thank R. Casalbuoni, P. Chiappetta, A. Deandrea, S. De Curtis, N. Di Bartolomeo, D. Dominici, R. Gatto, L. Maiani, A. Masiero for many stimulating discussions on the subject of these lectures. A special thank goes to the organizers of the Seminar, G. Marchesini and E. Onofri, as well as to the secretariat, for the very nice hospitality enjoyed in Parma. 


\section{References}

[1] F. Gursey, Nuovo Cim. 230 (1960) 230

[2] S. Weinberg, Physica 96A (1979) 327

[3] J. Gasser and H. Leutwyler, Ann. Phys. 158 (1984) 142; Nucl. Phys. B250 (1985) 465.

[4] H. Leutwyler, talk given at the XXVI Int. Conf. on High Energy Physics, Dallas, Aug. 1992, preprint BUTP-92/42.

[5] For a recent review see, for instance: G. Ecker, preprint CERN-TH.6660/92 and references therein

[6] B. Holdom and J. Terning, Phys. Lett. 247B (1990) 88

[7] M. Golden and L. Randall, Nucl. Phys. B361 (1991) 3

[8] H. Georgi, Nucl. Phys. B363 (1991) 301

[9] B. Holdom, Phys. Lett. B258 (1991) 156

[10] A. De Rujula, M.B. Gavela, P. Hernandez and E. Masso, preprint CERN-TH6272-91, FTUAM-91-31.

[11] T. Appelquist and C. Bernard, Phys. Rev. D22 (1980) 200

[12] A.C. Longhitano, Phys. Rev. D22 (1980) 1166; T. Appelquist in Gauge Theories and experiments at High Energies, ed. by K.C. Brower and D.G. Sutherland, Scottish University Summer School in Physics, St. Andrews (1980).

[13] A.C. Longhitano, Nucl. Phys. B188 (1981) 118

[14] M. Veltman, Acta Phys. Pol. B8 (1977) 475; B.W. Lee, C. Quigg and H.B. Thacker, Phys. Rev. D16 (1977) 1519; R. Casalbuoni, D. Dominici and R. Gatto, Phys. Lett. 147B (1984) 419; M.B. Einhorn, Nucl. Phys. B246 (1984) 75 .

[15] T. Appelquist and J. Carazzone, Phys. Rev. D11 (1975) 2856.

[16] E. D'Hoker and E. Fahri, Nucl. Phys. B248 (1984) 59; Nucl. Phys. B248 (1984) 77.

[17] G.L. Lin, H. Steger and Y.P. Yao, Phys. Rev. D44 (1991) 2139

[18] F. Feruglio, L. Maiani and A. Masiero, Nucl. Phys. B387 (1992) 523 
[19] E. D'Hoker, Phys. Rev. Lett. 69 (1992) 1316

[20] S.L. Adler, Phys. Rev. 177 (1969) 2426; J.S. Bell and R. Jackiw, Nuovo Cim. 60A (1969) 47; W.A. Bardeen, Phys. Rev. 184 (1969) 1848.

[21] J. Wess and B. Zumino, Phys. Lett. 37B (1971) 95; E. Witten, Nucl. Phys. B223 (1983) 422.

[22] S. Weinberg, Phys. Rev. D19 1277 (1979); L. Susskind, Phys. Rev. D20 (1979) 2619; E. Fahri and L. Susskind, Phys. Rep. 74 (1981) 279.

[23] S. Chadha and M. Peskin, Nucl. Phys. B185 (1981) 61; M. Peskin, Nucl. Phys. B175 (1980) 197; J. Preskill, Nucl. Phys. B177 (1981) 21; R. Casalbuoni, D. Dominici, S. De Curtis, N. Di Bartolomeo, F. Feruglio and R. Gatto, Phys. Lett. B285 (1992) 103.

[24] For a discussion in the framework of the electroweak interactions, see ref. [8].

[25] A. Manohar and H. Georgi, Nucl. Phys. B234 (1984) 189; H. Georgi and L. Randall, Nucl. Phys. B276 (1986) 241; D. Espriu, E. de Rafael and J. Taron, Nucl. Phys. B345 (1990) 22; H. Georgi, preprint HUTP-92/A036.

[26] A.P. Balachandran, A. Stern and G, Trahern, Phys. Rev. D19 (1979) 2416; M. Bando, T. Kugo and K. Yamawaki, Phys. Rep. 164 (1988) 217.

[27] M. Bando, T. Kugo, S. Uehara, K. Yamawaki and T. Yanagida, Phys. Rev. Lett. 54 (1985) 1215; see also G. Ecker, J. Gasser, A. Pich and E. de Rafael, Nucl. Phys. B231 (1989) 311.

[28] R. Casalbuoni, S. De Curtis, D. Dominici and R. Gatto, Phys. Lett. B155 (1985) 95; Nucl. Phys. B282 (1987) 235.

[29] R. Casalbuoni, P. Chiappetta, D. Dominici, F. Feruglio and R. Gatto, Nucl. Phys. B310 (1988) 181; A. Dobado and M. J. Herrero, Phys. Lett. B228 (1989) 495.

[30] S. Ferrara, A. Masiero and M. Porrati, preprint CERN-TH.6726/92 and NYUTH-92/11/02.

[31] E. Cremmer, B. Julia, J. Scherk, S. Ferrara, L. Girardello and P. van Niewenhuizen, Nucl. Phys. B147 (1979); E. Cremmer, S. Ferrara, L. Girardello and A van Proeyen, Nucl. Phys. B212 (1983) 413.

[32] L. Dixon, V. Kaplunovsky and J. Louis, Nucl. Phys. B355 (1991) 649; J-P. Deredinger, S. Ferrara, C. Kounnas and F. Zwirner, Nucl. Phys. B372 (1992) 145 and references therein. 
[33] S. Coleman, J. Wess and B. Zumino, Phys. Rev. 177 (1969) 2239; C. Callan, S. Coleman, J. Wess and B. Zumino, Phys. Rev. 177 (1969) 2247.

[34] R. Haag, Phys. Rev. 112 (1958) 669; D. Ruelle, Helv. Phys. Acta 35 (1962) 34; H.J. Borchers, Nuovo Cim. 25 (1960) 270.

[35] S. Coleman in Hadron and Their Interactions, ed. A. Zichichi (Academic Press. Inc.,New York, 1968)

[36] L. Rolandi, talk given at the XXVI Int. Conf. on High Energy Physics, Dallas, Aug. 1992, to appear in the proceedings.

[37] R.D. Peccei and X. Zhang, Nucl. Phys. B337 (1990) 269; R.D. Peccei, S. Peris and X. Zhang, Nucl. Phys. B349 (1991) 305; M. Frigeni, R. Rattazzi, Phys. Lett. B269 (1991) 412.

[38] M. Peskin and T. Takeuchi, Phys. Rev. Lett. 65 (1990) 964; W. Marciano and J. Rosner, Phys. Rev. Lett. 65 (1990) 2963; D. Kennedy and P. Langacker, Phys. Rev. Lett. 65 (1990) 2967; A. Ali and G. Degrassi, preprint DESY-91035; A. Dobado, D. Espriu, and M.J. Herrero, Phys. Lett. B255 (1991) 405; R.D. Peccei and S. Peris, Phys. Rev. D44 (1991) 809; J. Ellis, G.L. Fogli and E. Lisi, preprint CERN-TH-6363-92, BARI-TH-96-92.

[39] G. Altarelli and R. Barbieri, Phys. Lett. 253B (1991) 161;

[40] G. Altarelli, R. Barbieri and S. Jadach, Nucl. Phys. B369 (1992) 3.

[41] See for instance: M. Consoli, F. Jagerlehner and W. Hollik in Z Physics at LEP 1, Vol. 1, eds. G. Altarelli, R. Kleiss and C. Verzegnassi, CERN 89-08, Geneva, 1989

[42] G. Altarelli, talk given at the Rencontres de Moriond on Electroweak Interactions and Unified Theories, Les Arcs, France, March 15-22, 1992.

[43] H. Georgi, Nucl. Phys. B361 (1991) 339

[44] W. Buchmüller and D. Wyler, Nucl. Phys. B268 (1986) 621

[45] K. Hagiwara, R.D. Peccei, D. Zeppenfeld and K. Hikasa, Nucl. Phys. B282 (1987) 253.

[46] K. Hagiwara, S. Ishihara, R. Szalapski and D. Zeppenfeld, Phys. Lett. B283 (1992) 353;

[47] G. Valencia, talk given at the XXVI Int. Conf. on High Energy Physics, Dallas, Aug. 1992, FERMILAB-CONF-92/246-T 
[48] S. Bertolini and A. Sirlin, Nucl. Phys. B248 (1984) 589

[49] J.A. Bagger, Lectures given at the 1991 Theoretical Advanced Studies Institute, Boulder, Colorado, preprint JHU-TIPAC-910038. 